\title{
Kinematic instabilities in two-layer eccentric annular flows, part 2: shear-thinning and yield stress effects
}

\author{
M.A. Moyers-Gonzalez \\ Département de mathématiques et de statistique, Université de Montréal, CP 6128 \\ succ. Centre-Ville Montréal, QC, H3C 3J7, Canada. (moyers@dms.umontreal.ca) \\ I.A. Frigaard* \\ Department of Mathematics and Department of Mechanical Engineering, \\ University of British Columbia, 2054-6250 Applied Science Lane, Vancouver, BC, \\ V6T 1Z4, Canada. (frigaard@mech.ubc.ca)
}

August 31st, 2007

Abstract. This paper investigates the possibility of kinematic interfacial instabilities occurring during the industrial process of primary cementing of oil and gas wells. This process involves to flows in narrow eccentric annuli that are modelled via a Hele-Shaw approach. The fluids present in primary cementing are strongly non-Newtonian, usually exhibiting shear-thinning behaviour and often with a yield stress. The study is a sequel to [1] in which the base analysis has been developed for the case of 2 Newtonian fluids. The occurrence of static mud channels in primary cementing has been known of since the 1960's, [2], and is a major cause of process failure. We quantify this phenomenon, providing a simple semi-analytic expression for the maximal volume of residual fluid left behind in the annulus, $f_{\text {static }}$, and illustrate the dependency of $f_{\text {static }}$ on its five dimensionless parameters. We show that 3 of the 4 different types of static channel flows are linearly stable.

Via dimensional analysis, we show that the base flows depend on a minimal set of 8 dimensionless parameters and the stability problem depends on an additional 3 dimensionless parameters. This large dimensional parameter space precludes use of the full numerical solution to the stability problem as a predictive tool or for studying the various stability regimes. Instead we have developed a semi-analytical approach based on solution of the long wavelength limit. This stability results can be evaluated via simple quadrature from the base flow and is suitable for use in process optimisation.

Keywords: Primary cementing, multi-layer flow stability, Hele-Shaw flow, interfacial instability, kinematic instability, shear-thinning, yield stress

\section{Introduction}

This paper investigates the possibility of kinematic interfacial instabilities occurring during the industrial process of primary cementing of oil and gas wells. This process involves displacement flows of multiple fluids in narrow eccentric annuli, see Fig. 1, with the eventual aim of placing a cement slurry in uncontaminated state within the well,

* Corresponding author

(C) 2008 Kluwer Academic Publishers. Printed in the Netherlands. 

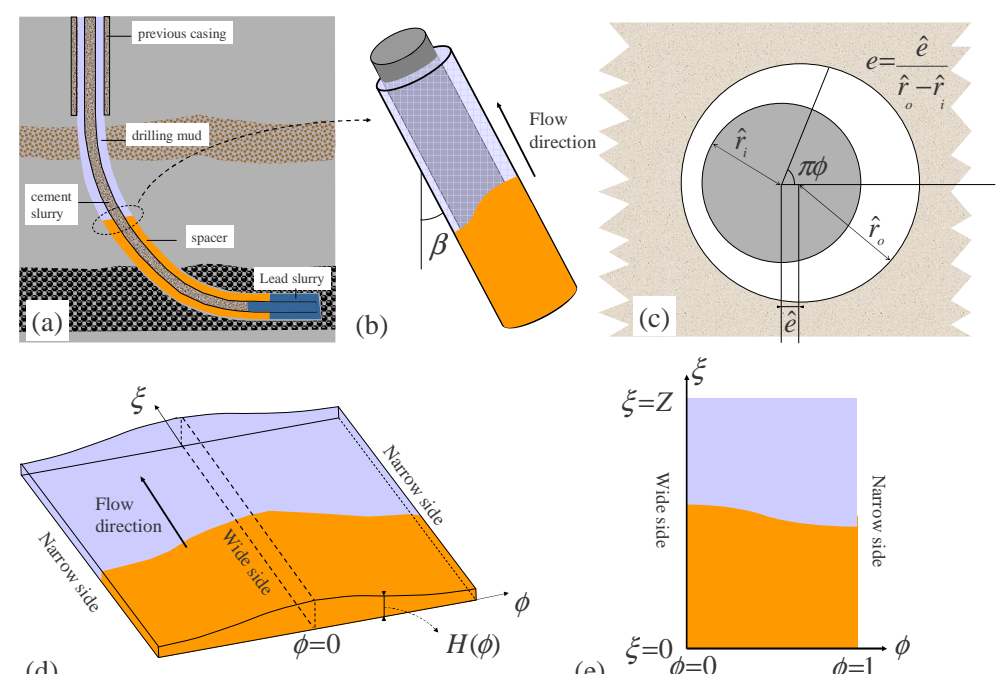

(d)

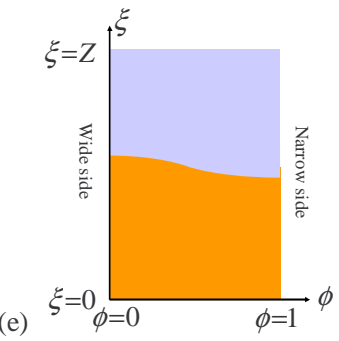

(f)
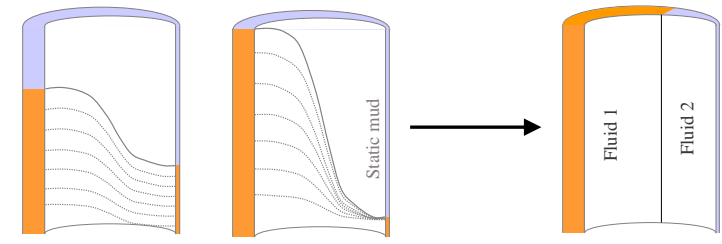

Figure 1. Cementing geometries: a) schematic of fluid stages pumped during a primary cementing operation; b) uniform section of eccentric annulus; c) eccentric annular cross-section; d) periodic eccentric annular Hele-Shaw cell; e) final computational domain, assuming symmetry at wide and narrow sides of the annulus; f) unsteady and static mud channel displacements that evolve into parallel flows.

between casing and formation where it may solidify to form a hydraulic seal. The process, its importance and some of the process problems that may occur are discussed in $[3,4,5]$, as well as more widely in the technical literature.

Of relevance to us is a process of unsteady fingering that occurs when the displacement front advances faster along the wide side of the eccentric annulus than the narrow side, possibly leaving behind a channel of drilling mud on the narrow (lower) side of the annulus, see e.g. $[2,6,7]$. Since sections of oil and gas wells that are cemented tend to be very long, by comparison with their azimuthal length-scale, the process of unsteady fingering and channeling results in flow regimes that are pseudo-parallel with the axis of the well, see Fig. 1f. The onset of this type of unsteady fingering is predicted by the analysis in [8] which is based on a Hele-Shaw modeling approach developed in 
$[9,10,11]$. Although the approach is a Hele-Shaw approach, it is worth commenting that the occurrence of unsteady interfaces that lead to pseudo-parallel flows (Fig. 1f) is not controlled by local mobility ratios as in classical Hele-Shaw instabilities. Here there is a global imbalance caused by the annular eccentricity, i.e. fluids flow faster in the wider part of the annulus, and also the annular flow rate is fixed, which constrains the local flow dynamics in a non-local fashion.

The aim of this paper is to study the stability of the parallel flows that evolve during displacements. Experimental evidence for such instabilities may be found in $[12,13]$. To study these flows we consider perturbations about a parallel steady state, aligned in the direction of the annulus. The base flows are thus steady solutions of a steady Darcy-flow problem with 2 fluids. In such flows, in the absence of surface tension the interface is simply advected with the flow. Although instability can be studied using a steady Darcy model and with time evolution confined to the kinematic equation, this approach assumes that any interfacial instability grows initially on the advective timescale. This may however not be true when the instabilities are instigated by either viscosity or density differences, or perhaps by a forced temporal perturbation, e.g. pulsation of a pump, (this latter possibility is not studied here). Therefore, in $[14,15]$ we have used a modified HeleShaw approach in which the linear acceleration terms are retained in the momentum balance. Evidently, should a linear instability grow then eventually the nonlinear inertial terms are also important. However, for predicting the onset of stability this approach suffices.

The study is a sequel to [1] (part 1) in which the base analysis has been developed for the case of 2 Newtonian fluids. The fluids present in primary cementing are however strongly non-Newtonian, usually exhibiting shear-thinning behaviour and often with a yield stress. This paper studies this practical reality, by extending the methodology of [1] to Herschel-Buckley fluids, which incorporate the simpler Bingham and power law fluid models. For brevity we refer the reader to part 1 for a proper introduction to the industrial problem area and a motivation for our study.

In part 1 we showed that the Newtonian stability problem depended on 7 dimensionless parameters and analysed this parametric dependency. Analytic solutions were developed for most of the limiting simplifications, e.g. concentric annuli, long wavelength limits. With such a high-dimensional parameter space the results in [1] contain significant complexity. Some effects on stability, such as the effect of including a positive or negative density difference in an inclined annulus, were physically intuitive but not all. 
Much of the complexity in the results arises from the competing effects of inertial accelerations and viscous dissipation. This is captured in the long wavelength analysis in [1], where the controlling parameter that switches from stable to unstable involves a product of the difference in interfacial velocities and a (weighted) difference in the Reynolds numbers, i.e. the kinematic viscosity difference. The interfacial velocities (capturing inertial effects) depend essentially on the viscosity difference. Thus, transitions between stable and unstable regimes show a strong dependency on the relative ratios of kinematic and dynamic viscosities, modulated by buoyancy. Part of the goal of the current paper is to see if the same overall picture is true once shear-thinning and yield stress effects are included.

The physical expectations of including shear-thinning are unclear. In general shear-thinning will reduce the effective viscosity, but here we work with an imposed flow rate so that the effects on the mean velocity are less apparent and will depend on the effective viscosity ratio. This is further complicated by the fact that at the same imposed mean velocity, the shear rate will be higher and the viscosity consequently lower in a narrower part of the annulus for a fluid that shear-thins. Global effects on the stability of the multi-layer flow are therefore difficult to predict.

For yield stress effects, to some extent physical intuition is easier. Increasing the yield stress will unequivocally increase the effective viscosity. However a new phenomena arises: that of the fluids becoming "stuck" in the narrow annulus. This problematic phenomenon has been recognised in the cementing industry for at least 40 years, [2], and is a major cause of failure of primary cementing. In the context of our problem, we have multi-layer flows that contain a static unyielded channel, running axially along the well axis. Here we look at the effects of these channels on stability.

An outline of the paper and the chief results are as follows. Section 2 describes the extension of the model from part 1 to non-Newtonian fluids, and an analysis of the base flows. In particular in $\S 2.2$ we analyse the static mud channel phenomenon, clarifying its dependency on dimensionless parameters and giving predictions of the maximal static residual annular volume. Section 3 presents the linear stability problem and reduces the dependency of the problem to a minimal set of dimensionless numbers. A number of simplified analyses, leading to analytic and semi-analytical results are presented in $\S 4$. Section 5 presents numerical results, focusing in two subsections on shear-thinning and yield stress effects. For the shear-thinning fluids for vertical annuli the results are ambiguous. For horizontal annuli the flow is stabilised by positive buoyancy and slightly destabilised by shear-thinning effects. The yield stress has the general effect of suppressing short wavelength 
instabilities. For sufficiently large yield stress part of the annulus can become static. In cases where the static channel abuts the interface these flows are shown to be stable. We also show two new type of transition in our results: from stable to static channel and direct from unstable to static channel.

\section{Non-Newtonian displacement flows}

In this paper we shall consider the stability of base flows of two nonNewtonian fluids in a narrow eccentric annulus. The underlying HeleShaw model is derived in $[14,15]$, see also Fig. 1 for a schematic of the geometry. In [1] we have considered the simpler problem of stability of the parallel flow of two Newtonian fluids. Therefore, we keep our description minimalist to avoid undue repetition. As in part 1 we work in an unwrapped half-annular domain $\Omega:(\phi, \xi) \in[0,1] \times[-\infty, \infty]$, which we assume is divided into two fluid domains, $\Omega_{1}$ and $\Omega_{2}$, by a smooth curve $C$ that is defined by a level set function:

$$
F(\phi, \xi, t)=0 .
$$

The function $F(\phi, \xi, t)$ satisfies the gap-averaged kinematic equation:

$$
\frac{H}{\epsilon} \frac{\partial F}{\partial t}-\frac{\partial \Psi}{\partial \xi} \frac{\partial F}{\partial \phi}+\frac{\partial \Psi}{\partial \phi} \frac{\partial F}{\partial \xi}=0
$$

where $H(\phi)=1+e \cos \pi \phi$ denotes the gap width, $\epsilon$ is the ratio of advective and viscous timescales, and $\Psi(\phi, \xi, t)$ is the gap-averaged stream function. The annular eccentricity is $e$ and the annulus is inclined at angle $\beta$ to the vertical: see Fig. 1b \& c. In general all variables we use will be dimensionless. When dimensional variables are defined they will be denoted with a $\hat{\imath}$ symbol.

The physical properties of each fluid are assumed constant in each domain, and the stream function formulation of the Darcy-like equations gives us that

$$
\rho_{k} \nabla \cdot\left[\frac{\nabla \Psi_{t}}{H}\right]+\nabla \cdot \mathbf{S}_{k}=0, \quad(\phi, \xi) \in \Omega_{k}, k=1,2,
$$

in each fluid domain. The vectorfield $\mathbf{S}_{k}$ contains the components of modified pressure gradient in fluid $k$. As the Hele-Shaw approach is taken, the modified pressure gradient is related to mean velocity by an hydraulic relation that can be calculated locally by considering the flow of the fluid between parallel plates, see e.g. [9]. In general, $\mathbf{S}_{k}$ will 
have form:

$$
\begin{array}{r}
\mathbf{S}_{k}=\left(\frac{\chi_{k}(|\nabla \Psi|)+\tau_{k, Y} / H}{|\nabla \Psi|}\right) \nabla \Psi \Leftrightarrow\left|\mathbf{S}_{k}\right|>\tau_{k, Y} / H, \\
|\nabla \Psi|=0 \Leftrightarrow\left|\mathbf{S}_{k}\right| \leq \tau_{k, Y} / H .
\end{array}
$$

Here $\tau_{k, Y}$ denotes the yield stress of fluid $k$. We assume that our fluids are shear-thinning yield stress fluids, modeled for simplicity by the Herschel-Bulkley model, each characterised by $\tau_{k, Y}$, a dimensionless consistency $\kappa_{k}$ and a power-law index $n_{k}$. The function $\chi$ is a positive increasing function of $|\nabla \Psi|$, which represents the purely viscous part of the frictional pressure gradient. The exact form of $\chi$ depends on $H$, and on the rheological parameters that characterise the fluid. For the Herschel-Bulkley model, $\chi$ is defined implicitly by the relation:

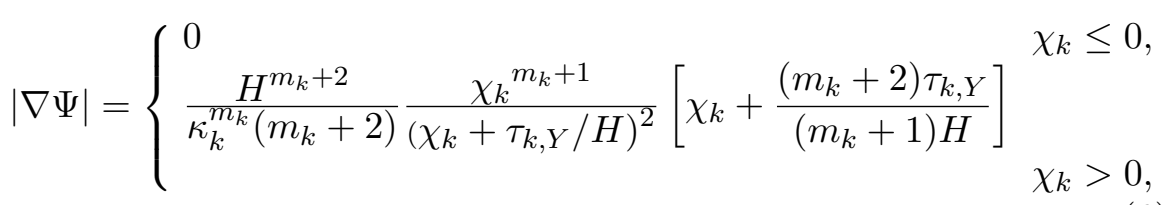

where $m_{k}=1 / n_{k}$.

To give a brief physical explanation of the above model (2)-(6), first note that the gap-averaged velocity field in directions $(\phi, \xi)$ is simply $\left(-\Psi_{\xi}, \Psi_{\phi}\right) / H$, and so (2) simply says that the interface is advected with the mean flow. In deriving (3) the usual Hele-Shaw steps have been followed except that we have chosen to work with the viscous timescale instead of the advective timescale. Typically one assumes the process is steady and discards all the inertial terms with the argument that the Reynolds number times the aspect ratio (gap/length) of the duct is small. As discussed in the introduction, here we look for instabilities that may arise from a combination of density \& viscosity differences, or (later) from flow oscillation. In these cases it is not clear that the only timescale is the advective timescale and hence we have retained also the time derivatives. If instabilities grow then of course neglect of the nonlinear inertial terms is questionable, but for now the objective is to predict onset. The other difference with some Hele-Shaw treatments is that we have chosen to work with the stream function formulation rather than the pressure formulation. This is because for yield stress fluids the pressure gradient is indeterminate in unyielded flow regions. To return to something familiar for the reader, if we set $\tau_{Y}=0$, then (6) becomes explicit:

$$
\chi_{k}=\kappa_{k}\left[\frac{\left(m_{k}+2\right)|\nabla \Psi|}{H^{m_{k}+2}}\right]^{1 / m_{k}},
$$


which leads to the familiar linear relation for $m_{k}=1$, (i.e. for Newtonian fluids: see part 1), and on neglecting the time derivatives in (3) we have a linear elliptic equation familiar form $2 \mathrm{D}$ porous media flows.

Equation (3) is supplemented with the following 2 boundary conditions:

$$
\begin{aligned}
& \Psi(0, \xi, t)=0 . \\
& \Psi(1, \xi, t)=1,
\end{aligned}
$$

on the wide $(\phi=0)$ and narrow $(\phi=1)$ sides of the annulus, respectively. The physical interpretation is simply that the flow rate through the annulus is fixed, with $\Psi(1, \xi, t)=1$ in (8) arising from the scaling of the velocity with the mean velocity. At the interface between fluids the following two jump/continuity conditions are satisfied,

$$
\begin{aligned}
& \left.\Psi\right|_{1} ^{2}=0, \\
& {\left.\left[\left(\frac{\rho_{k} \cos \beta}{S t^{*}}, \frac{\rho_{k} \sin \beta \sin \pi \phi}{S t^{*}}\right)+\frac{\rho_{k}}{H} \nabla \Psi_{t}+\mathbf{S}_{k}\right] \cdot \nabla F\right|_{1} ^{2}=0 .}
\end{aligned}
$$

The first of these is simply continuity of $\Psi$, i.e. of the normal velocity, whereas (10) defines the jump in normal derivative of $\Psi$. Condition (10) comes from continuity of the pressure, see $[14,1]$ for further explanation.

For a given interface, we observe that these unsteady displacement flows are characterised by 12 dimensionless parameters. These parameters are: two dynamic parameters, $\epsilon$ and $S t^{*}$; two geometric parameters, $e$ and $\beta$; four dimensionless physical parameters for each fluid, $\rho_{k}, \kappa_{k}$, $\tau_{k, Y}, n_{k}, k=1,2$. Although complex, we shall see later that there is some redundancy in the parameter space, when considering specific aspects of the flow. We shall discuss the dimensionless parameters in more detail in $\S 3.2$.

\subsection{BASE TWO-LAYER FLOWS}

We study the stability of steady multi-layer flows, that arise as one fluid fingers past another during an annular displacement at unit flow rate. These steady base flows satisfy (2)-(10) and are parallel to the $\xi$ axis, hence $\Psi=\Psi(\phi)$. We assume that the annulus is occupied by two fluids: fluid 1 occupying $\phi \in\left[0, \phi_{i}\right)$ and fluid 2 occupying $\phi \in\left(\phi_{i}, 1\right]$. The interface level set is thus $F=\phi-\phi_{i}$. Our solutions are steady parallel solutions of (3), which implies $\boldsymbol{S}_{k}=\left(S_{k, \phi}, S_{k, \xi}\right)=\left(S_{k, \phi}, 0\right)$, and

$$
\frac{\partial}{\partial \phi} S_{k, \phi}=0
$$


Since we also have:

$$
S_{k, \phi}=-\frac{\partial p}{\partial z}-\frac{\rho_{k} \cos \beta}{S t^{*}},
$$

with (10) this implies that the axial pressure gradient is continuous at the interface. Therefore the fluid layers are acted on by a constant pressure gradient, modified by an axial hydrostatic pressure that is different in each fluid layer, due to the density jump. Since $S_{k, \phi}$ is independent of $\phi$ and $\xi$, (by definition, we look for solutions independent of $\xi$ ), we write:

$$
S_{1, \phi}=A, \quad S_{2, \phi}=A-b,
$$

where $b$ is a buoyancy parameter, given by:

$$
b=\frac{\rho_{2}-\rho_{1}}{S t^{*}} \cos \beta .
$$

Typically, in a cementing scenario where fingering occurs, $b$ is negative: the heavier cement channels past the more viscous drilling mud that is left behind on the narrow side. The constant $A$ represents the modified pressure gradient in the axial direction, within fluid 1, and must be found as part of the base solution. In order to find $A$ we use (8):

$$
1=\Psi(1)=\left.\int_{0}^{\phi_{i}} \frac{\partial \Psi}{\partial \phi}\right|_{k=1} \mathrm{~d} \phi+\left.\int_{\phi_{i}}^{1} \frac{\partial \Psi}{\partial \phi}\right|_{k=2} \mathrm{~d} \phi
$$

where,

$$
\begin{aligned}
\left.\frac{\partial \Psi}{\partial \phi}\right|_{k=1} & =\Upsilon\left(\frac{A}{\kappa_{1}}, \frac{\tau_{1, Y}}{\kappa_{1}}, H, m_{1}\right), \\
\left.\frac{\partial \Psi}{\partial \phi}\right|_{k=2} & =\Upsilon\left(\frac{A-b}{\kappa_{2}}, \frac{\tau_{2, Y}}{\kappa_{2}}, H, m_{2}\right) \\
\Upsilon(w, x, y, z) & =\left\{\begin{array}{l}
0, \\
\operatorname{sgn}(w) \frac{y^{2}(|w| y-x)^{z+1}}{|w| y(z+2)}\left(1+\frac{x}{|w| y(z+1)}\right), \\
|w| y>x,
\end{array}\right.
\end{aligned}
$$

c.f. equation (6). Note that $\phi$-dependency enters through $H(\phi)$.

Equation (15) can be interpreted is a nonlinear equation for $A / \kappa_{1}$. It is straightforward to show that the function $\Upsilon(w, x, y, z)$ increases monotonically with $w$, and from this we find that (15) has a unique solution $A / \kappa_{1}=A\left(\phi_{i}\right) / \kappa_{1}$. Once $A\left(\phi_{i}\right) / \kappa_{1}$ is determined, the base solution can be computed via integration. More precisely, we have that the base solution will depend parametrically on $\phi_{i}, e, \kappa_{1} / \kappa_{2}, b / \kappa_{2}$, $\tau_{1, Y} / \kappa_{1}, m_{1}, \tau_{2, Y} / \kappa_{2}, m_{2}$. By comparison with part 1 , where we considered Newtonian fluids, $m_{k}=1$, we see that we have 4 additional 
dimensionless parameters to consider, i.e. $\tau_{1, Y} / \kappa_{1}, m_{1}, \tau_{2, Y} / \kappa_{2}, m_{2}$. The ratios $\tau_{k, Y} / \kappa_{k}$ will be denoted $B_{k}$, and are the Bingham numbers for each fluid, which represent the balance of yield and viscous stresses.

Some example of base solution are shown below in Fig. 2. In Fig. 2a we have no buoyancy force; $\Psi(\phi)$ increases from 0 to 1 with $\phi$, but there is a discontinuity in the gradient at the interface, $\phi=\phi_{i}$. Recall that the derivative of $\Psi(\phi)$ divided by $H(\phi)$ gives the axial velocity. In a HeleShaw cell at an interface between fluids, only the pressure and normal velocity are conserved. Fig. $2 \mathrm{~b}$ shows a base solution for which fluid 2 is static in a region close to $\phi=1$ and Fig. 2c shows a base flow for which the entire layer of fluid 2 is static. This phenomena results from the yield stress in fluid 2 being too large. In the context of the industrial displacement flow, this would correspond to the drilling mud being left behind in a static channel on the narrow side of the annulus, which is detrimental to the process effectiveness. We study these static channels in the section immediately following. Finally, Fig. 2d illustrates that with significant buoyancy, the base flow velocity can be in the negative direction, i.e. the flow can be counter-current.

\subsection{Static Fluid LAYERS}

We have seen in Fig. $2 \mathrm{~b} \& \mathrm{c}$ that it is possible for fluid 2 to be stationary, in all or part of $\left(\phi_{i}, 1\right]$. It is also possible for all or part of the fluid 1 layer to be static. In the context of flow stability, we shall later see that certain of these base flows are linearly stable and therefore it is of interest to define where these flows can be found. From the practical perspective, it is however the fluid 2 static layers that are of interest and which we therefore focus on.

Physically, a static channel occurs when the modified pressure gradient is not large enough to overcome the yield stress of the fluid. Since the modified pressure gradient is constant in each fluid layer and since $H(\phi)$ decreases, yield stress fluids become static at the largest values of $\phi$ in each layer. For the mud, fluid 2, the minimal annular gap is at $\phi=1$, where $H=1-e$, and so there can only be a static "mud" channel in fluid 2 if

$$
\left|A\left(\phi_{i}\right)-b\right|<\frac{\tau_{2, Y}}{1-e} .
$$

If (19) is satisfied, the entire layer will be static if

$$
\left|A\left(\phi_{i}\right)-b\right|<\frac{\tau_{2, Y}}{H\left(\phi_{i}\right)}
$$

and otherwise $\left[\phi_{s}, 1\right] \subset\left(\phi_{i}, 1\right]$ is static, where $\left|A\left(\phi_{i}\right)-b\right|=\tau_{2, Y} / H\left(\phi_{s}\right)$. The fully static channels are of industrial relevance, since they represent the residual mud that may possibly remain in the wellbore after 

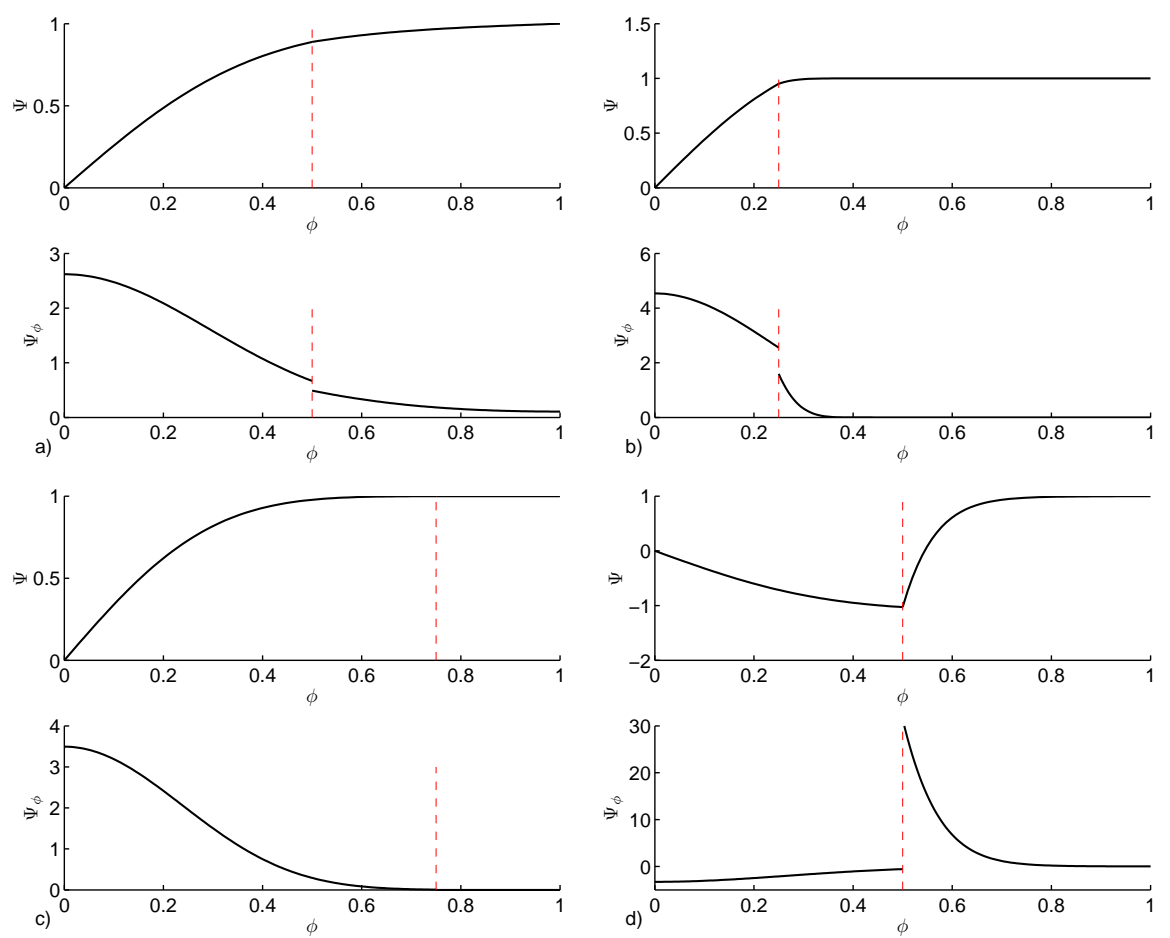

Figure 2. Example base solutions: a) $\phi_{i}=0.5, e=0.3, \kappa_{1} / \kappa_{2}=1, b / \kappa_{2}=0$, $B_{1}=1, m_{1}=2, B_{2}=1, m_{2}=1$; b) $\phi_{i}=0.25, e=0.75, \kappa_{1} / \kappa_{2}=2, b / \kappa_{2}=-1.25$, $B_{1}=0.25, m_{1}=2, B_{2}=5, m_{2}=5$; c) $\phi_{i}=0.75, e=0.75, \kappa_{1} / \kappa_{2}=2$, $b / \kappa_{2}=-1.25, B_{1}=0.25, m_{1}=2, B_{2}=5, m_{2}=5$; d) $\phi_{i}=0.5, e=0.5$, $\kappa_{1} / \kappa_{2}=0.5, b / \kappa_{2}=-5, B_{1}=0.25, m_{1}=2, B_{2}=1, m_{2}=5$. Interface position is marked by the vertical broken line.

cementing. Suppose now that (20) is satisfied for some $\phi_{i}$. We now ask what is the maximal azimuthal width of fully static mud channel that can exist?

First of all, if the mud is static, then the modified pressure gradient is determined wholly from the fluid 1 layer, via:

$$
1=\left.\int_{0}^{\phi_{i}} \frac{\partial \Psi}{\partial \phi}\right|_{k=1} \mathrm{~d} \phi
$$

From our previous discussion, we see that the modified pressure gradient $A\left(\phi_{i}\right) / \kappa_{1}$ will depend on $\phi_{i}, e, \tau_{1, Y} / \kappa_{1}$ and $m_{1}$. Evidently as $\phi_{i} \rightarrow 0$, if the mud remains static, then $A\left(\phi_{i}\right) / \kappa_{1} \rightarrow \infty$ since the unit flow rate is forced through an increasingly narrow azimuthal gap. It follows that for some $\phi_{i}$, there will be a value of $A\left(\phi_{i}\right)$ for which:

$$
\left|A\left(\phi_{i}\right)-b\right|=\frac{\tau_{2, Y}}{H\left(\phi_{i}\right)}
$$



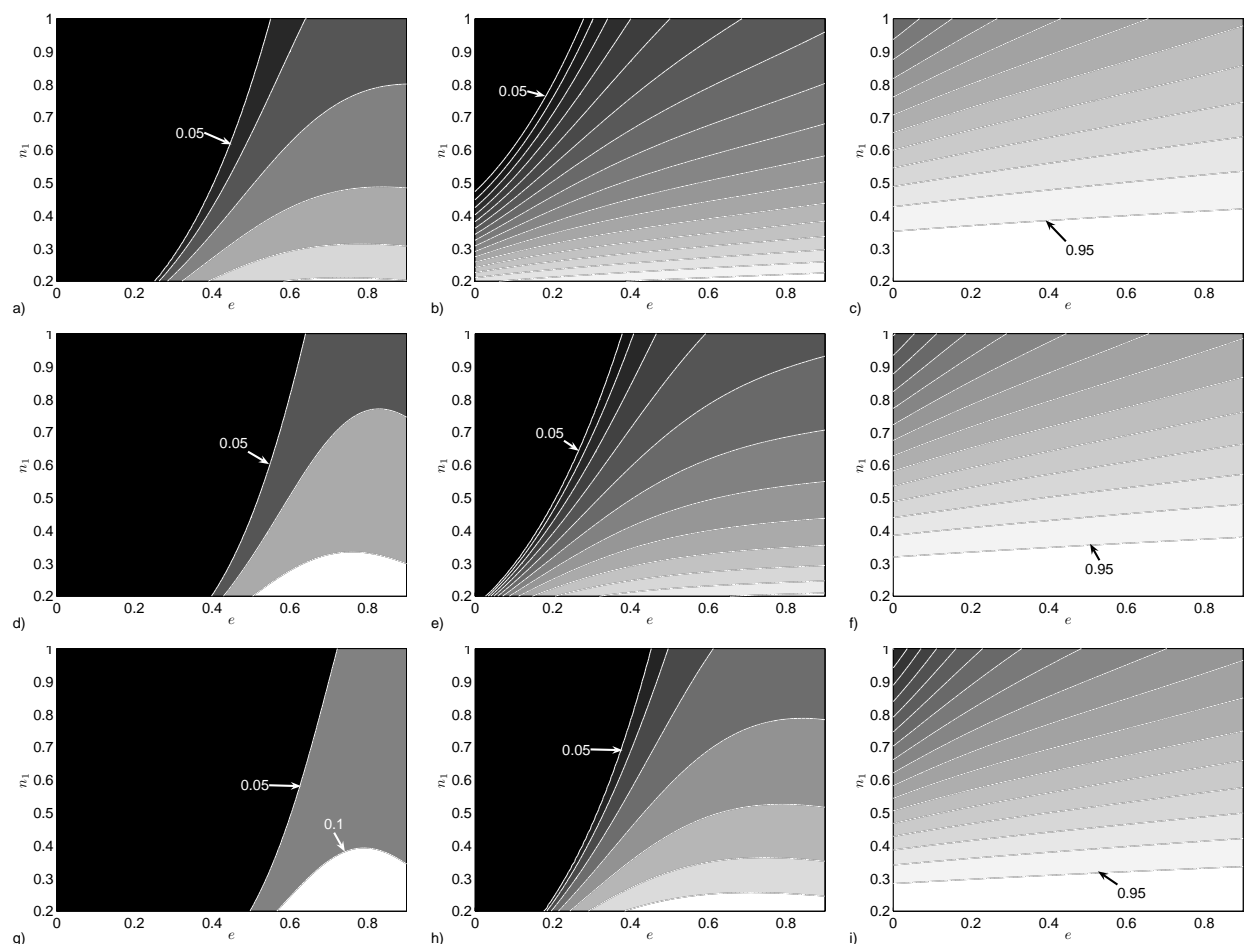

Figure 3. Variations in maximal displaced fluid volume left static in the annulus, $f_{\text {static }}$ with $b=0$ : a) $B_{1}=0, \tau_{2, Y} / \kappa_{1}=1$; b) $B_{1}=0, \tau_{2, Y} / \kappa_{1}=2$; c) $B_{1}=0$, $\tau_{2, Y} / \kappa_{1}=5$; d) $B_{1}=0.5, \tau_{2, Y} / \kappa_{1}=1$; e) $B_{1}=0.5, \tau_{2, Y} / \kappa_{1}=2$; f) $B_{1}=0.5$, $\tau_{2, Y} / \kappa_{1}=5$; g) $B_{1}=1, \tau_{2, Y} / \kappa_{1}=1$; h) $B_{1}=1, \tau_{2, Y} / \kappa_{1}=2$; i) $B_{1}=1, \tau_{2, Y} / \kappa_{1}=5$. Shaded contours are spaced at intervals of $\Delta f_{\text {static }}=0.05$.

which defines the smallest interface position for which the mud layer remains fully static. We denote this interface position by $\phi_{i}=\phi_{i, \min }$, and the maximal azimuthal width of mud channel is therefore $1-\phi_{i, \min }$. Dividing through by $\kappa_{1}$, we see that $\phi_{i, \min }$ depends parametrically on: $e, B_{1}, m_{1}, b / \kappa_{1}$ and $\tau_{2, Y} / \kappa_{1}$. More useful practically is to know the maximal volume of the annulus occupied by static mud, say $f_{\text {static }}$ :

$$
f_{\text {static }}=\int_{\phi_{i, m i n}}^{1} H(\phi) \mathrm{d} \phi=1-\phi_{i, \min }-\frac{e}{\pi} \sin \pi \phi_{i, \min } .
$$

In Figs. $3 \& 4$ we explore some of this parametric variation.

In general we see that the maximal residual mud fraction, $f_{\text {static }}$, increases with the ratio $\tau_{2, Y} / \kappa_{1}$ and decreases with $B_{1}$ and with $n_{1}$. The former is a simple increase in the stress level required to mobilise fluid 2 , and therefore is wholly intuitive. The decrease with $B_{1}$ and with $n_{1}$ is also unsurprising, as these increase the viscous stress generated in fluid 1 , which is transmitted to fluid 2 via the axial pressure gradient. 

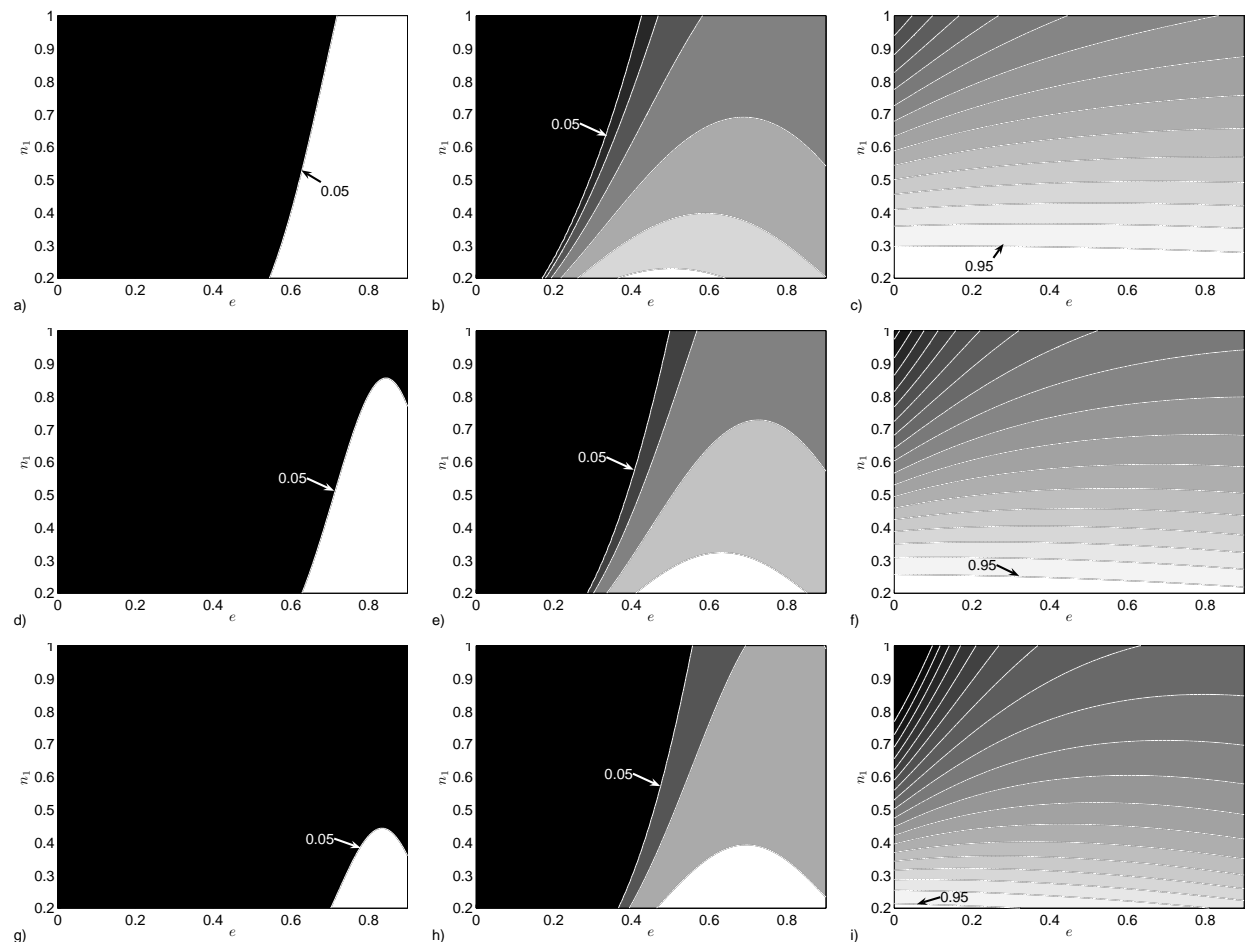

Figure 4. Variations in maximal displaced fluid volume left static in the annulus, $f_{\text {static }}$ with $b=-1$ : a) $B_{1}=0, \tau_{2, Y} / \kappa_{1}=1$; b) $B_{1}=0, \tau_{2, Y} / \kappa_{1}=2$; c) $B_{1}=0$, $\tau_{2, Y} / \kappa_{1}=5$; d) $B_{1}=0.5, \tau_{2, Y} / \kappa_{1}=1$; e) $B_{1}=0.5, \tau_{2, Y} / \kappa_{1}=2 ;$ f) $B_{1}=0.5$, $\tau_{2, Y} / \kappa_{1}=5$; g) $B_{1}=1, \tau_{2, Y} / \kappa_{1}=1$; h) $B_{1}=1, \tau_{2, Y} / \kappa_{1}=2$; i) $B_{1}=1, \tau_{2, Y} / \kappa_{1}=5$. Shaded contours are spaced at intervals of $\Delta f_{\text {static }}=0.05$.

Usually we find that $f_{\text {static }}$ increases with the eccentricity $e$, and it is only at low $n_{1}$ that some non-monotone behaviour is found. The precise reasons for the non-monotone behaviour are unclear. Finally, comparing Figs. $3 \& 4$ directly, we see that reducing $b$ has the effect of decreasing $f_{\text {static }}$. In summary, most of these effects are physically intuitive.

A similar analysis can be carried out for the possibility of fluid 1 static layers. The only difference to note is that if fluid 1 is static anywhere in the fluid layer, it is static at the interface which is the narrowest part of the annulus occupied by fluid 1 .

\section{Stability of parallel two-layer flows}

As in part 1, [1], we consider linear perturbation of the base flows from $\S 2.1$, which we denote by $\Psi_{k}=\Psi_{k, 0}(\phi) \& \mathbf{S}_{k}=\left(S_{k, \phi, 0}, 0\right), k=1,2$, 
with interface position $\phi_{i}=\phi_{i, 0}$. We linearise, assume normal mode expansions and transform to an eigenvalue problem, in the usual way. More formally, for $\delta \ll 1$ we assume the expansions:

$$
\begin{aligned}
\Psi_{k} & =\Psi_{k, 0}+\delta \Psi_{k, 1}+\delta^{2} \Psi_{k, 2}+\ldots \\
\mathbf{S}_{k} & =\left(S_{k, \phi, 0}, 0\right)+\delta \mathbf{S}_{k, 1}+\delta^{2} \mathbf{S}_{k, 2}+\ldots \\
\phi_{i} & =\phi_{i, 0}+\delta h+\ldots
\end{aligned}
$$

substitute into (2)-(10), expand in powers of $\delta$.

$$
\begin{aligned}
\nabla \Psi_{k} & =\left(\Psi_{k, 0, \phi}+\delta \Psi_{k, 1, \phi}+\ldots, \delta \Psi_{k, 1, \xi}+\ldots\right) \\
|\nabla \Psi| & =\left[\left(\Psi_{k, 0, \phi}+\delta \Psi_{k, 1, \phi}+\ldots\right)^{2}+\left(\delta \Psi_{k, 1, \xi}+\ldots\right)^{2}\right]^{\frac{1}{2}} \\
& \sim\left|\Psi_{k, 0, \phi}\right|+\delta \operatorname{sgn}\left(\Psi_{k, 0, \phi}\right) \Psi_{k, 1, \phi}+O\left(\delta^{2}\right) .
\end{aligned}
$$

When the fluid is yielded we find:

$$
\mathbf{S}_{k, 1}=\left[\chi_{k}^{\prime}\left(\left|\Psi_{k, 0, \phi}\right|\right) \Psi_{k, 1, \phi},\left(\chi_{k}\left(\left|\Psi_{k, 0, \phi}\right|\right)+\frac{\tau_{k, Y}}{H}\right) \frac{\Psi_{k, 1, \xi}}{\left|\Psi_{k, 0, \phi}\right|}\right],
$$

but otherwise $\mathbf{S}_{k}$ is indeterminate. The linearised equation for $\Psi_{1}$ is

$$
\nabla \cdot\left[\mathbf{S}_{k, 1}+\frac{\rho_{k}}{H} \nabla \Psi_{k, 1, t}\right]=0, \quad \text { in } \Omega_{k} .
$$

The kinematic equation and boundary conditions are:

$$
\begin{aligned}
\frac{H}{\epsilon} h_{t}+\Psi_{k, 1, \xi}+\Psi_{k, 0, \phi} h_{\xi} & =0, \quad \text { at } \phi=\phi_{i, 0} \\
\Psi_{1,1}(0, \xi, t) & =0, \\
\Psi_{2,1}(1, \xi, t) & =0,
\end{aligned}
$$

The jump conditions (9) \& (10) are linearised, first about the basic flow and secondly onto the basic flow interface position. Thus, at $O(\delta)$, condition (9) becomes

$$
\left.\left(\Psi_{k, 1}+h \Psi_{k, 0, \phi}\right)\right|_{k=1} ^{k=2}=0, \quad \text { at } \phi=\phi_{i, 0},
$$

and we note that it is the derivative of this quantity with respect to $\xi$ that appears in (27), i.e. it is irrelevant which fluid is considered for the kinematic condition. Expanding $\left(\mathbf{S}_{k}+\left(\rho_{k} / H\right) \nabla \Psi_{k, t}\right) \cdot \mathbf{n}$, about $\phi=\phi_{i, 0}$ we have:

$$
\begin{aligned}
& {\left[\mathbf{S}_{k}+\frac{\rho_{k}}{H} \nabla \Psi_{k, t}\right] \cdot \mathbf{n} \sim } S_{k, 0, \phi}+\delta h \frac{\partial}{\partial \phi} S_{k, 0, \phi}+\delta S_{k, 1, \phi} \\
&+\frac{\rho_{k}}{H}\left[\Psi_{k, 0, \phi t}+\delta h \Psi_{k, 0, \phi \phi t}+\delta \Psi_{k, 1, \phi t}\right] \\
& \text { at } \phi=\phi_{i, 0} \\
&\left(1, \tan \beta \sin \pi \phi_{i}\right) \cdot \mathbf{n} \sim 1-\delta h_{\xi} \tan \beta \sin \pi \phi_{i, 0}
\end{aligned}
$$


Noting that $S_{k, 0, \phi}$ is independent of $\phi$ and $\Psi_{k, 0}$ independent of $t$, at $O(\delta)$, condition $(10)$ becomes

$$
\left.\left(S_{k, 1, \phi}+\frac{\rho_{k}}{H} \Psi_{k, 1, \phi t}\right)\right|_{k=1} ^{k=2}=b h_{\xi} \tan \beta \sin \pi \phi_{i, 0}, \quad \text { at } \phi=\phi_{i, 0},
$$

We now assume a normal mode expansion of the linear perturbation, with modes of form:

$$
\Psi_{1, k} \sim f_{k}(\phi) e^{i(\alpha \xi-s t)}, \quad h \sim h_{0} e^{i(\alpha \xi-s t)},
$$

which we substitute into (26)-(31) to give:

$$
\begin{aligned}
0= & D\left[\chi_{1}^{\prime}\left(\left|\Psi_{1,0, \phi}\right|\right) D f_{1}\right]-\alpha^{2}\left[\frac{\chi_{1}\left(\left|\Psi_{1,0, \phi}\right|\right)+\frac{\tau_{1, Y}}{H}}{\left|\Psi_{1,0, \phi}\right|}\right] f_{1}, \\
& -i s \rho_{1}\left(D\left(\frac{D}{H}\right)-\frac{\alpha^{2}}{H}\right) f_{1}, \quad \phi \in\left(0, \phi_{i, 0}\right) \\
0= & D\left[\chi_{2}^{\prime}\left(\left|\Psi_{2,0, \phi}\right|\right) D f_{2}\right]-\alpha^{2}\left[\frac{\chi_{2}\left(\left|\Psi_{2,0, \phi}\right|\right)+\frac{\tau_{2, Y}}{H}}{\left|\Psi_{2,0, \phi}\right|}\right] f_{2}, \\
& -i s \rho_{2}\left(D\left(\frac{D}{H}\right)-\frac{\alpha^{2}}{H}\right) f_{2}, \\
f_{1}(0)= & 0 \\
f_{2}(1)= & 0
\end{aligned}
$$

and at $\phi=\phi_{i, 0}$ :

$$
\begin{aligned}
-\frac{H}{\epsilon} s h_{0}+\alpha\left(f_{k}+\Psi_{k, 0, \phi} h_{0}\right) & =0 \\
\left.\left(h_{0} \Psi_{0, k, \phi}+f_{k}\right)\right|_{1} ^{2} & =0 \\
{\left.\left[\left(\chi_{k}^{\prime}\left(\left|\Psi_{0, k, \phi}\right|\right)-i s \frac{\rho_{k}}{H}\right) D f_{k}\right]\right|_{k=1} ^{k=2} } & =i \alpha b h_{0} \tan \beta \sin \pi \phi_{i, 0},
\end{aligned}
$$

Assuming for the moment that both fluid layers are fully mobile, linear stability of this flow is governed by the system (32)-(38), which is an an eigenvalue problem for $s=s_{R}+\mathrm{i} s_{I} \in \mathbb{C}$. Linear instability is found if for any $\alpha \in \mathbb{R}$ we have an eigenvalue with $s_{I}>0$. We may observe that, including specification of $\alpha$ and $\phi_{i, 0}$, this eigenvalue problem for $s$ depends upon 14 dimensionless parameters. 


\subsection{Reduction to a minimal SET of Dimensionless PARAMETERS}

We are able to simplify the stability problem by dividing through (32) by $\kappa_{1},(33)$ by $\kappa_{2}$ and (38) by $\kappa_{2}$. With the following definitions:

$$
\begin{array}{rlrl}
\mathrm{B}_{1} & =\frac{\tau_{1, Y}}{\kappa_{1}}, & \mathrm{~B}_{2} & =\frac{\tau_{2, Y}}{\kappa_{2}}, \\
\operatorname{Re}_{1} & =\frac{\epsilon \rho_{1}}{\kappa_{1}}, & \operatorname{Re}_{2}=\frac{\epsilon \rho_{2}}{\kappa_{2}}, \\
\varphi_{\kappa} & =\frac{\kappa_{1}}{\kappa_{2}} & \varphi_{b} & =\frac{b}{\kappa_{2}} \\
\lambda & =\frac{s}{\epsilon}, & &
\end{array}
$$

eigenvalues $\lambda \in \mathbb{C}$ are found from the solution to:

$$
\begin{aligned}
0= & D\left[\frac{\chi_{1}^{\prime}\left(\left|\Psi_{1,0, \phi}\right|\right)}{\kappa_{1}} D f_{1}\right]-\alpha^{2}\left[\frac{\left|A / \kappa_{1}\right|}{\left|\Psi_{1,0, \phi}\right|}\right] f_{1}, \\
& -i \lambda \operatorname{Re}_{1}\left(D\left(\frac{D}{H}\right)-\frac{\alpha^{2}}{H}\right) f_{1}, \quad \phi \in\left(0, \phi_{i, 0}\right) \\
0= & D\left[\frac{\chi_{2}^{\prime}\left(\left|\Psi_{1,0, \phi}\right|\right)}{\kappa_{2}} D f_{2}\right]-\alpha^{2}\left[\frac{\left|\varphi_{\kappa} A / \kappa_{1}-\varphi_{b}\right|}{\left|\Psi_{2,0, \phi}\right|}\right] f_{2}, \\
& -i \lambda \operatorname{Re}_{2}\left(D\left(\frac{D}{H}\right)-\frac{\alpha^{2}}{H}\right) f_{2}, \quad \phi \in\left(\phi_{i, 0}, 1\right) \\
f_{1}(0)= & 0 \\
f_{2}(1)= & 0
\end{aligned}
$$

and at $\phi=\phi_{i, 0}$ :

$$
\begin{aligned}
-H \lambda h_{0}+\alpha\left(f_{k}+\Psi_{k, 0, \phi} h_{0}\right)= & 0, \\
\left.\left(h_{0} \Psi_{0, k, \phi}+f_{k}\right)\right|_{1} ^{2}= & 0, \\
{\left[\frac{\chi_{2}^{\prime}\left(\left|\Psi_{1,0, \phi}\right|\right)}{\kappa_{2}}-\mathrm{i} \frac{\lambda \operatorname{Re}_{2}}{H}\right] D f_{2}=} & \varphi_{\kappa}\left[\frac{\chi_{1}^{\prime}\left(\left|\Psi_{1,0, \phi}\right|\right)}{\kappa_{1}}-\mathrm{i} \frac{\lambda \operatorname{Re}_{1}}{H}\right] D f_{1} \\
& +\mathrm{i} \alpha \varphi_{b} h_{0} \tan \beta \sin \pi \phi_{i, 0},
\end{aligned}
$$

Recall that the base solution $\Psi_{k, 0}$ is fully determined by the 8 parameters: $\phi_{i, 0}, e, \varphi_{\kappa}, \varphi_{b}, \mathrm{~B}_{1}, m_{1}, \mathrm{~B}_{2}, m_{2}$, which also determine $A / \kappa_{1}$ above. Note also from the base solution that:

$$
|A|=\chi_{1}\left(\left|\Psi_{1,0, \phi}\right|\right)+\frac{\tau_{1, Y}}{H}, \quad|A-b|=\chi_{2}\left(\left|\Psi_{2,0, \phi}\right|\right)+\frac{\tau_{2, Y}}{H} .
$$


We must add to this set of 8 parameters the two reduced Reynolds numbers, $\operatorname{Re}_{1} \& \operatorname{Re}_{2}$. Finally, we may note from (6) that $\chi_{k}^{\prime}\left(\left|\Psi_{k, 0, \phi}\right|\right)$ is defined via implicit differentiation of (6):

$$
\chi_{k}^{\prime}=\frac{\kappa_{k}^{m_{k}}\left(m_{k}+2\right)}{H^{m_{k}+2} \chi_{k}^{m_{k}}}\left[\frac{\left(\chi_{k}+\tau_{k, Y} / H\right)^{3}}{m \chi_{k}^{2}+\frac{m_{k}\left(m_{k}+2\right) \chi_{k} \tau_{k, Y}}{\left(m_{k}+1\right) H}+\frac{\tau_{k, Y}^{2}}{\left(m_{k}+1\right) H^{2}}}\right],
$$

from which we observe that $\chi_{k}^{\prime} / \kappa_{k}$ depends on $\phi_{i, 0}, e, \mathrm{~B}_{k}$ and $m_{k}$, plus $\chi_{k} / \kappa_{k}$. Now we have that $\chi_{1} / \kappa_{1}=\left|A / \kappa_{1}\right|-\mathrm{B}_{1} / H$ and $\chi_{2} / \kappa_{2}=$ $\left|\varphi_{\kappa} A / \kappa_{1}-\varphi_{b}\right|-\mathrm{B}_{2} / H$, and these depend also on the parameters of the base solution. Thus including $\alpha$, we have reduced from 14 to 11 dimensionless parameters.

\subsection{Dimensionless nUmbers}

Our final stability problem remains formidable in terms of the 11dimensional parameter space that we must consider. However, first we discuss the dimensionless parameters in relation to the dimensional physical parameters of the process. The reader is also referred to part 1 , where much of this is explained. First of all, we consider a two-fluid flow through a narrow eccentric annulus of eccentricity $e$ and angle of inclination $\beta ; \phi_{i, 0}$ has a similarly simple geometric interpretation.

The mean axial speed of the fluids (recall the flow rate is imposed) is denoted $\hat{w}^{*}$ and $\hat{\rho}^{*}$ denotes the maximum of the two fluid densities, (recall our convention of denoting dimensional quantities with the $\hat{*}$ symbol). A shear rate scale $\hat{\gamma}^{*}$ is defined by

$$
\hat{\dot{\gamma}}^{*}=\frac{\hat{w}^{*}}{\hat{r}_{o}-\hat{r}_{i}}
$$

where $\hat{r}_{o} \& \hat{r}_{i}$ are the outer and inner radii of the annulus, respectively. This is used to define a shear stress scale:

$$
\hat{\tau}^{*}=\max _{k=1,2}\left\{\hat{\tau}_{k, Y}+\hat{\kappa}_{k}\left(\hat{\dot{\gamma}}^{*}\right)^{n_{k}}\right\}
$$

and from this a viscosity scale $\hat{\mu}^{*}=\hat{\tau}^{*} / \hat{\dot{\gamma}}^{*}$. These are used to scale the dimensional rheological parameters: $\kappa_{k}=\hat{\kappa}_{k}\left(\hat{\dot{\gamma}}^{*}\right)^{n_{k}} / \hat{\tau}^{*}, \tau_{k, Y}=\hat{\tau}_{k, Y} / \hat{\tau}^{*}$, and hence we have that

$$
\mathrm{B}_{k}=\frac{\hat{\tau}_{k, Y}}{\hat{\kappa}_{k}\left(\hat{\dot{\gamma}}^{*}\right)^{n_{k}}}
$$


The Bingham numbers thus denote the ratio of yield stress to purely viscous stress in each fluid. The power law indices, $n_{k}=1 / m_{k}$, describe the shear-thinning properties of the fluids; typically $n_{k} \leq 1$. The viscosity ratio $\varphi_{\kappa}=\kappa_{1} / \kappa_{2}$ is seen to be the ratio of purely viscous shear stresses in the two fluids. With respect to part 1, where we have considered only Newtonian fluids, the above definitions collapse to the Newtonian definitions in the case that the yield stresses are zero $\left(\mathrm{B}_{k}=0\right)$ and $n_{k}=1$. Thus, essentially we have only 4 additional parameters to consider.

The two Reynolds numbers, $\operatorname{Re}_{k}, \quad k=1,2$ are the non-Newtonian counterparts of those defined in part 1 :

$$
\operatorname{Re}_{k}=\frac{\hat{\rho}_{k} \hat{w}^{*}\left(\hat{r}_{o}-\hat{r}_{i}\right)^{2}}{\pi \hat{r}_{a} \hat{\kappa}_{k}\left(\hat{\dot{\gamma}}^{*}\right)^{n_{k}-1}}
$$

where $\hat{r}_{a}=0.5\left(\hat{r}_{o}-\hat{r}_{i}\right)$ is the mean radius. These are reduced Reynolds numbers, relevant to the Hele-Shaw scaling, (i.e. the usual Reynolds number multiplied by the aspect ratio). Typically we would expect that $\operatorname{Re}_{k} \lesssim 1$.

The buoyancy parameter $\varphi_{b}$ is defined by:

$$
\varphi_{b}=\frac{\left[\hat{\rho}_{2}-\hat{\rho}_{1}\right] \hat{g}\left(\hat{r}_{o}-\hat{r}_{i}\right) \cos \beta}{\hat{w}^{*} \hat{\kappa}_{2}\left(\hat{\dot{\gamma}}^{*}\right)^{n_{2}}},
$$

which is a ratio between the axial buoyancy stresses and the viscous stresses in fluid 2. Use of the axial buoyancy stresses above results in the $\tan \beta$ term appearing in (49), which becomes singular for horizontal wells, $\beta=\pi / 2$. Therefore, at times it will be convenient to work instead with

$$
\varphi_{b}^{*}=\frac{\left[\hat{\rho}_{2}-\hat{\rho}_{1}\right] \hat{g}\left(\hat{r}_{o}-\hat{r}_{i}\right)}{\hat{w}^{*} \hat{\kappa}_{2}\left(\hat{\dot{\gamma}}^{*}\right)^{n_{2}}} .
$$

Regarding the wavenumber $\alpha$, note that the lengthscale used in the dimensionless model is $\pi \hat{r}_{a}$, and thus wavelengths of instabilities, $2 \pi / \alpha$, need to be scaled appropriately. Using the eigenvalue $\lambda$ will give an equivalent stability characterization to that using $c$, but time is measured in terms of the advective timescale, $\pi \hat{r}_{a} / \hat{w}^{*}$.

\section{Simplified analyses}

A number of simplified cases may be treated, for which analytical progress is possible. 


\subsection{Stability Of Single Fluid FLOWS}

First of all, let us suppose that we have the flow of a single fluid in the annulus. The stability problem is as above, but setting e.g. $\phi_{i, 0}=1$, suppressing the interface conditions, fluid 2 layer and subscripts. This leaves:

$$
\begin{aligned}
0= & D\left[\frac{\chi^{\prime}\left(\left|\Psi_{0, \phi}\right|\right)}{\kappa} D f\right]-\alpha^{2}\left[\frac{|A / \kappa|}{\left|\Psi_{0, \phi}\right|}\right] f, \\
& -i \lambda \operatorname{Re}\left(D\left(\frac{D}{H}\right)-\frac{\alpha^{2}}{H}\right) f, \quad \phi \in(0,1) \\
f(0)= & 0, \\
f(1)= & 0 .
\end{aligned}
$$

On multiplying by the complex conjugate $f^{*}$ and integrating over $[0,1]$, it is straightforward to show that $\lambda_{I}<0$ and therefore the single fluid flow is linearly stable.

\subsection{Stability of 2 LAYER FlOWS With STATIC CHANNELS}

In considering 2-fluid flows, in which one or both fluids have a static channel, we may also simplify things. First note that there are 4 different types of flow: (i) fluid 1 is fully static; (ii) fluid 1 is partly static; (iii) fluid 2 is fully static; (iv) fluid 2 is partly static. In addition there are combinations of these 4 possibilities.

Suppose that fluid $k$ contains a static channel. If the entire fluid layer is static, then due to the eccentricity of the annulus this implies that the stress is below the yield stress by a finite amount everywhere in the layer (except perhaps at the widest part of the fluid layer, where it may be at exactly the yield stress). In this case, the infinitesimal linear perturbation cannot generate a finite stress perturbation and hence the velocity and stream function perturbation is zero: $f_{k}=D f_{k}=0$. According to the kinematic condition, either $h_{0}=0$ or $\lambda=0$, implying neutral stability. However with $h_{0}=0$, the interface is not perturbed and the stability problem in the other fluid layer becomes that of a single fluid, i.e. linearly stable by the method in $\S 4.1$ above. Therefore, the flows (i) \& (iii) are linearly stable.

Let us consider (ii), where fluid 1 is partly static. Due to eccentricity, the mobile region is the wider part of the annulus so that we have mobile fluid 1 for $\phi \in\left[0, \phi_{s}\right)$ and static fluid for $\phi \in\left[\phi_{s}, \phi_{i}\right)$, for some $\phi_{s}$. In considering perturbation of the solution and a possible perturbation to the yield surface, $\phi_{s}$, after carefully linearising about $\phi_{s}$ we find that

$$
D f_{1}\left(\phi_{s}\right)=\alpha f_{1}\left(\phi_{s}\right)=0 .
$$


We now multiply $(43)$ by $f_{1}^{*}$ and integrate across $\left[0, \phi_{s}\right]$ in the manner of $\S 4.1$. This gives that either $f_{1}=0$ or $\lambda_{I}<0$. Taking $f_{1}=0$, we may note that the interface is not perturbed. Therefore, $f_{2}\left(\phi_{i}\right)=0$, we may multiply (44) by $f_{2}^{*}$ and integrate across $\left[\phi_{i}, 1\right]$, to show that $f_{2}=0$ or $\lambda_{I}<0$. This establishes that any flow of partially yielded fluid 1 will also be linearly stable. Physically, this occurs because the interface is static and cannot deform, which means that the stability problems in the yielded fluid layers are completely decoupled \& treatable as single fluid problems.

Finally consider situation (iv), where fluid 2 is partially yielded. Instability can only arise if fluid 1 is fully mobile in $\left[0, \phi_{i, 0}\right)$, and since the fluid layer is widest at $\phi=\phi_{i, 0}$, fluid 2 is also mobile at the interface. The static fluid channel is now say $\left[\phi_{s}, 1\right]$. As before the static fluid channel is not perturbed, but now interfacial perturbations are possible. The full stability problem must be solved, but the domain for (44) is the mobile fluid layer: $\phi \in\left(\phi_{i, 0}, \phi_{s}\right)$, and (46) is replaced by

$$
D f_{2}\left(\phi_{s}\right)=\alpha f_{2}\left(\phi_{s}\right)=0 .
$$

Note that an additional boundary condition is required to resolve the singular behaviour in (44) as $\phi \rightarrow \phi_{s}$.

\subsection{Concentric annuli}

For concentric annuli, $(H=1)$, the base solution will have constant velocity in each fluid layer, so that (43) \& (44) reduce to constant coefficient equations. After long but straightforward manipulations, $\lambda$ is found to satisfy:

$$
\begin{aligned}
0= & \mathrm{i} \alpha \varphi_{b} \tan \beta \sin \pi \phi_{i, 0}+\varphi_{\kappa}\left[\frac{\chi_{1}^{\prime}}{\kappa_{1}}-\mathrm{i} \lambda \operatorname{Re}_{1}\right]\left[\lambda-\alpha W_{i, 1}\right] \nu_{1} \operatorname{coth} \nu_{1} \alpha \phi_{i, 0} \\
& +\left[\frac{\chi_{2}^{\prime}}{\kappa_{2}}-\mathrm{i} \lambda \operatorname{Re}_{2}\right]\left[\lambda-\alpha W_{i, 2}\right] \nu_{2} \operatorname{coth} \nu_{2} \alpha\left(1-\phi_{i, 0}\right)
\end{aligned}
$$

where

$$
\nu_{1}=\sqrt{\frac{\frac{\left|A / \kappa_{1}\right|}{\left|W_{i, 1}\right|}-\mathrm{i} \lambda \operatorname{Re}_{1}}{\frac{\chi_{1}^{\prime}}{\kappa_{1}}-\mathrm{i} \lambda \operatorname{Re}_{1}}}, \quad \nu_{2}=\sqrt{\frac{\frac{\left|\varphi_{\kappa} A / \kappa_{1}-\varphi_{b}\right|}{\left|W_{i, 2}\right|}-\mathrm{i} \lambda \mathrm{Re}_{2}}{\frac{\chi_{2}^{\prime}}{\kappa_{2}}-\mathrm{i} \lambda \operatorname{Re}_{2}}}
$$

and where the $W_{i, k}$ are the interfacial velocities of the base flow:

$$
W_{i, k}=\frac{1}{H\left(\phi_{i, 0}\right)} \frac{\mathrm{d}}{\mathrm{d} \phi} \Psi_{k, 0}\left(\phi_{i, 0}\right), \quad k=1,2 .
$$


For a concentric annulus, $H=1$ and these are also the constant velocities of each fluid layer.

Although we have been able to write down the above algebraic expression, it cannot be directly solved for $\lambda$, since $\lambda$ appears also in $\nu_{k}$. For the Newtonian fluids in part 1, the pressure gradient-flow rate relation is linear, which leads to $\nu_{k}=1$ and a quadratic equation for $\lambda$. Here the only practical use appears to be as a test condition for numerical solutions.

\subsection{LONG WAVELENGTH ANALYSIS}

The long wavelength limit, $\alpha \rightarrow 0$, is studied by assuming a regular perturbation expansion in $\alpha$ for both $\lambda$ and the solution to (43)-(49). After considerable algebra we find the following expressions:

$$
\begin{aligned}
\lambda \sim & \alpha \lambda_{1}+\alpha^{2} \lambda_{2}+O\left(\alpha^{3}\right) \\
\lambda_{1}= & \frac{M_{1} W_{i, 2}+M_{2} W_{i, 1}}{M_{1}+M_{2}} \\
\lambda_{2}= & \mathrm{i}\left\{-\frac{\varphi_{b} \tan \beta \sin \pi \phi_{i, 0}}{3 \pi \varphi_{\kappa} H\left(\phi_{i, 0}\right)}\left[\frac{M_{1} M_{2}}{M_{1}+M_{2}}\right]\right. \\
& \left.+\lambda_{1} \frac{\left(W_{i, 2}-W_{i, 1}\right)}{3}\left[\frac{\operatorname{Re}_{1} N_{1} M_{2}-\operatorname{Re}_{2} N_{2} M_{1}}{\left(M_{1}+M_{2}\right)^{2}}\right]\right\}
\end{aligned}
$$

where

$$
\begin{aligned}
& M_{1}=3 \pi \int_{0}^{\phi_{i, 0}} \frac{\kappa_{1}}{\chi_{1}^{\prime}\left(\left|\Psi_{1,0, \phi}\right|(\phi)\right)} \mathrm{d} \phi, \\
& M_{2}=3 \pi \varphi_{\kappa} \int_{\phi_{i, 0}}^{1} \frac{\kappa_{2}}{\chi_{2}^{\prime}\left(\left|\Psi_{2,0, \phi}\right|(\phi)\right)} \mathrm{d} \phi, \\
& N_{1}=9 \pi \int_{0}^{\phi_{i, 0}} \frac{\kappa_{1}^{2}}{H(\phi)\left[\chi_{1}^{\prime}\left(\left|\Psi_{1,0, \phi}\right|(\phi)\right)\right]^{2}} \mathrm{~d} \phi, \\
& N_{2}=9 \pi \varphi_{\kappa} \int_{\phi_{i, 0}}^{1} \frac{\kappa_{2}^{2}}{H(\phi)\left[\chi_{2}^{\prime}\left(\left|\Psi_{2,0, \phi}\right|(\phi)\right)\right]^{2}} \mathrm{~d} \phi .
\end{aligned}
$$

These expressions reduce to those for two Newtonian fluids, (as given in part 1), in the limit $m_{k}=1$ and $\mathrm{B}_{k}=0$.

It is interesting to observe that the same structure persists for the non-Newtonian fluid combinations as for the Newtonian fluids. The first order eigenvalue is advective (i.e. real), and propagates at a weighted average interfacial speed without growth or decay. The second order eigenvalue is complex and controls the stability. As in part 1 we can see that $\rho_{2}>\rho_{1}(\Rightarrow b>0)$ tends to stabilise the flow. Typically we 
will have $\lambda_{1}>0$ and then the second term in (60) is governed by the sign of:

$$
\left(W_{i, 2}-W_{i, 1}\right)\left(\operatorname{Re}_{1} N_{1} M_{2}-\operatorname{Re}_{2} N_{2} M_{1}\right),
$$

so that we can expect some form of checker-boarding when viewed in terms of interfacial velocity differences and Reynolds number differences.

\section{Numerical results}

For the remainder of the paper we consider parametric variations in the stability of the system of (43)-(49). Two tools are used for this. First, we solve numerically the full problem. Second, we consider the long wavelength limit, $\alpha \rightarrow 0$. The long wavelength limit gives sufficient conditions for linear instability, which is what we typically would like to predict. However, there are also some restrictions to the method, which we illustrate below.

The main part of our results we shall group in $\S 5.2 \& \S 5.3$, exploring shear-thinning and yield stress effects, respectively. Evidently, with an 11-dimensional parameter space to explore it is not possible to give a complete description of the stability. Our approach is to highlight the main qualitative features and illustrate this with concrete examples.

\subsection{Numerical SOlution OF the EigenVAlue PROBlem}

The system (43)-(49) is solved using a spectral method. These are global methods that use the fully discretized stability operator, which is supplied to a matrix eigenvalue solver to give the spectrum. We have chosen a Chebyshev polynomial expansion to discretize our problem. The use of Chebyshev polynomials, especially in bounded domains, has proven very effective and accurate for such problems. The implementation of the method for our problem is fairly standard, as described in [1]. The boundary conditions are handled via the method described in [16], which maps spurious eigenvalues to a fixed point in the complex plane, outside of the domain of interest. As in [1] the numerical spectra consist of two interfacial modes and two viscous spectra (each centered on the imaginary axis). Typically we have used $N=51$ polynomials for each fluid layer, which is adequate since usually the most unstable mode is one of the interfacial modes, i.e. increasing $N$ adds further viscous modes but does not significantly change the position of the interfacial modes or the largest viscous modes.

Numerically, 2 eigenvalues are mapped spuriously with the boundary condition implementation, which leaves 2 interfacial modes and $N-2$ 
viscous modes for each layer. Note that the viscous spectra are discrete and the eigenfunctions regular. The problem is thus quite different to some non-Newtonian stability problems, (e.g. with visco-elastic fluids), where continuous spectra/singular eigenfunctions are found. Here the non-Newtonian effects simply manifest in (parametrically) different data functions within the linear equations and boundary conditions. The underlying mathematical problem is not qualitatively changed from the Newtonian problem. An exception to this might be thought to occur when we have a yield stress fluid in the annulus. In this case $\chi_{k}^{\prime}$ and $1 /|\nabla \Psi|$ become singular at the yield surface. However, in the case that we have a yield surface, additional compatibility conditions are also satisfied by the stream function perturbation there, which render the perturbation equations regular. Details regarding this are given in [14] and a similar example of such problems is found in the study of shear instabilities for visco-plastic fluids, where again the inclusion of a yield stress does not bring about continuous spectra, see e.g. [17].

As a test problem for our numerical method, we use the analytic expression for the eigenvalues in a concentric annulus, (56). We denote the least stable eigenvalue by $\lambda_{\max }=\lambda_{R, \max }+\mathrm{i} \lambda_{I, \max }$, i.e. that for which $\lambda=\lambda_{R}+\mathrm{i} \lambda_{I}$ has maximal imaginary part. A typical computed spectrum for a concentric annulus is shown in Fig. 5a, for parameters $\beta=0, \varphi_{\kappa}=2.5, \varphi_{b}^{*}=-1, \phi_{i, 0}=0.5, e=0.0, m_{1}=1, B_{1}=2, B_{2}=1$, $\operatorname{Re}_{1}=1$ and $\operatorname{Re}_{2}=0.5$. For the concentric annulus the $\mathrm{N}-1$ viscous modes have coalesced/collapsed for each fluid into a single point, (with slight spreading of the spectra). For comparison, Fig. 5b shows the spectrum for the same parameters with a small eccentricity, $e=0.1$, where we observe that the viscous spectra have expanded. As a further test for our numerical method we substitute the numerical eigenvalues for the concentric annulus into (56) and take the absolute value of the residual, denoted $\left|\lambda_{e}\right|^{2}$. Table I shows these values for the parameters of Fig. 5. We may observe that, relative to the size of the eigenvalues, the errors are very small. These results are quite typical.

\subsection{Shear-thinning EFfects}

In order to explore parametric variations in some sort of systematic fashion, we utilise the long wavelength approximation, which provides sufficient conditions for flow instability. We fix the interface position at mid-azimuth, $\phi_{i, 0}=0.5$, and consider first vertical annuli $\beta=0$. Fig. 6 shows the effects of increasing $m_{2}$ at small eccentricity, i.e. we make the fluid that occupies the narrow side of the annulus progressively more shear-thinning. Fig. 7 shows the same results but at a high eccentricity, $e=0.5$. 

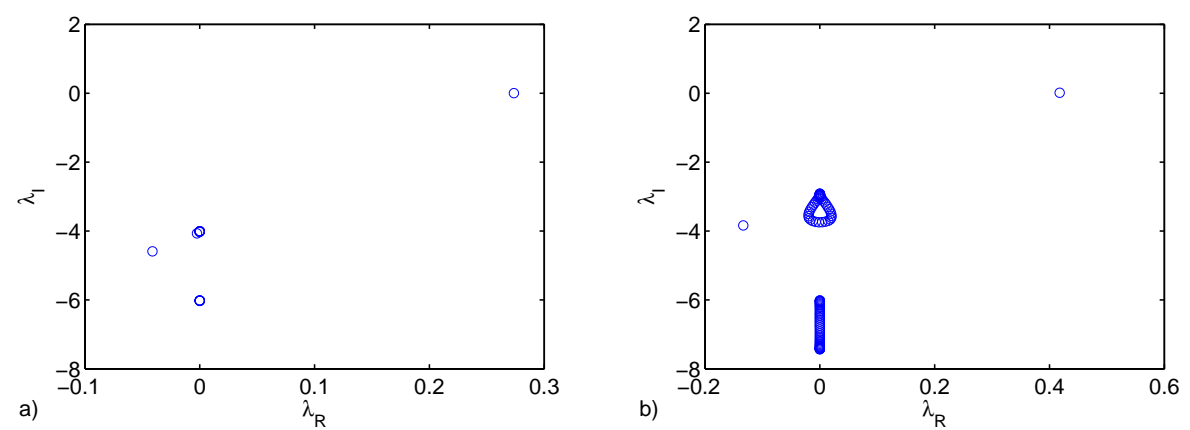

Figure 5. Spectrum for system (43)-(49): $\beta=0, \varphi_{\kappa}=2.5, \varphi_{b}^{*}=1, \phi_{i, 0}=0.5$, $m_{1}=1, B_{1}=2, B_{2}=1, \operatorname{Re}_{1}=1$ and $\operatorname{Re}_{2}=0.5$ : a) $e=0.0$; b) $e=0.1$.

Table I. Test of the numerical method. Numerical values of $\lambda$ substituted into (56) to give the residual error, $\left(\lambda_{e}\right)$. We present results for the largest of the viscous modes (which are clustered about the analytical solution) and the two interfacial modes.

$\begin{array}{cc}\text { Numerical } \lambda & \left|\lambda_{e}\right|^{2} \\ 3.559844652988294 \mathrm{e}-001+5.466326043265693 \mathrm{e}-003 \mathrm{i} & 2.964029969411872 \mathrm{e}-011 \\ -6.287673021974180 \mathrm{e}-002-4.238254642625869 \mathrm{e}+000 \mathrm{i} & 1.875940044675446 \mathrm{e}-020 \\ -1.716556341567676 \mathrm{e}-003-3.724625951864712 \mathrm{e}+000 \mathrm{i} & 1.074113563660485 \mathrm{e}-013 \\ -2.148646745047909 \mathrm{e}-006-6.021629304112192 \mathrm{e}+000 \mathrm{i} & 5.973585198654020 \mathrm{e}-009\end{array}$

We have plotted our results in the $(x, y)$ plane where

$$
x=\frac{\kappa_{1} / \kappa_{2}-1}{\kappa_{1} / \kappa_{2}+1}, \quad y=d\left(\operatorname{Re}_{2}, \operatorname{Re}_{1}, e\right)=\frac{\operatorname{Re}_{2} N_{2} M_{1}-\operatorname{Re}_{1} N_{1} M_{2}}{\left(M_{1}+M_{2}\right)^{2}} .
$$

Here $x$ is simply a nonlinear stretching of the consistency ratio, whereas $d\left(\operatorname{Re}_{2}, \operatorname{Re}_{1}, e\right)$ enters directly into the stability criterion (60). The consistency ratio tends to affect the interfacial velocity difference, which also appears in (60). Indeed, for fixed well inclination the product $\left(W_{i, 2}-W_{i, 1}\right) d\left(\operatorname{Re}_{2}, \operatorname{Re}_{1}, e\right)$ determines the long wave instability. Thus, the checkerboard of stable and unstable regimes in Figs. $6 \& 7$ is to be expected, as for $\beta=0$ buoyancy does not influence the long-wave instability (60). On the face of it, increased eccentricity does not result in a more or less stable flow, but simply a shifting in the checkerboard pattern.

Figs. $8 \& 9$ show the same results as Figs. $6 \& 7$, except that the variation of power law index for fluid 1 is explored, again at $e=0.1$ and $e=0.5$. Again the results are ambiguous, viewed in terms of what might be the natural questions to ask, i.e. if $m_{1}$ is increased does the flow become more or less stable? Figs. $8 \& 9$ demonstrate that the either 

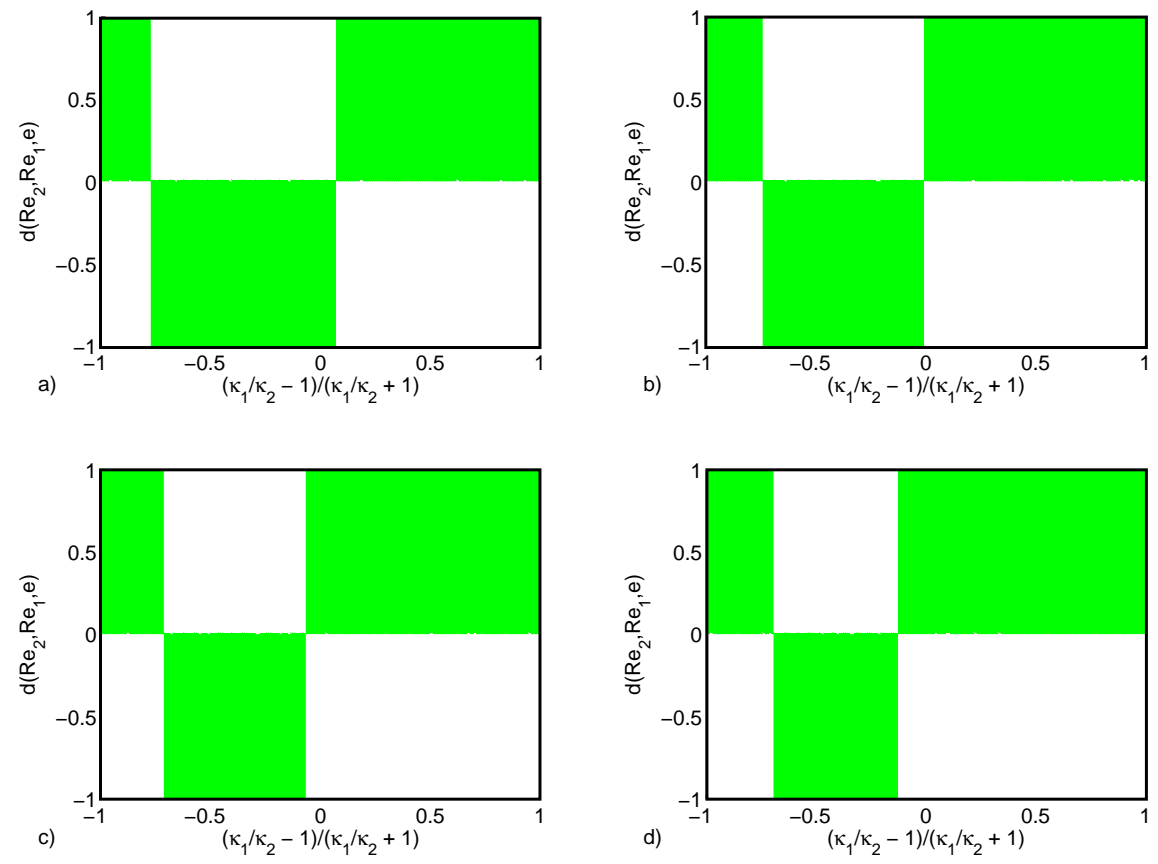

Figure 6. Regions of marginal stability, long wavelength approximation: $\beta=0$, $\varphi_{b}^{*}=1, \phi_{i, 0}=0.5, e=0.1, m_{1}=1, B_{1}=B_{2}=0$ : a) $m_{2}=4 / 3$; b) $m_{2}=2$; c) $m_{2}=3$; d) $m_{2}=5$; unstable - white, stable - green (light grey).
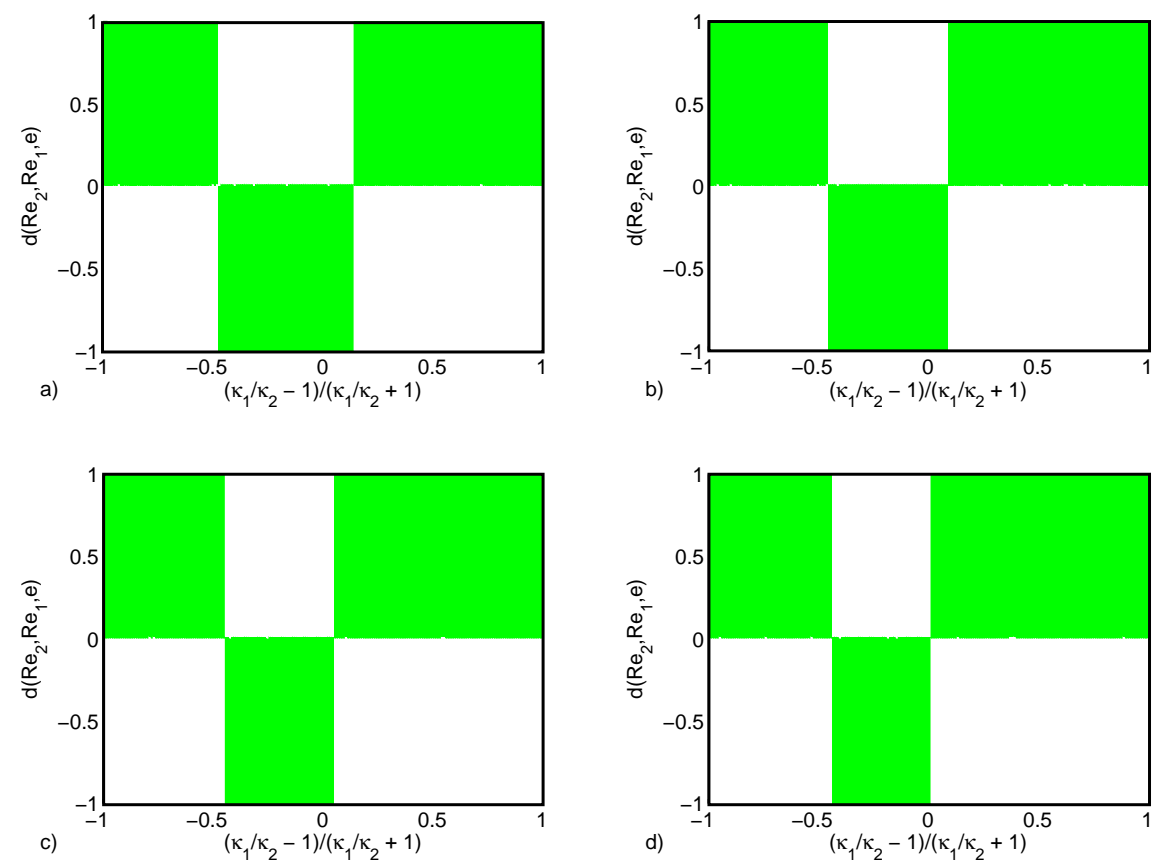

Figure 7. As Fig. 6 with $e=0.5$. 

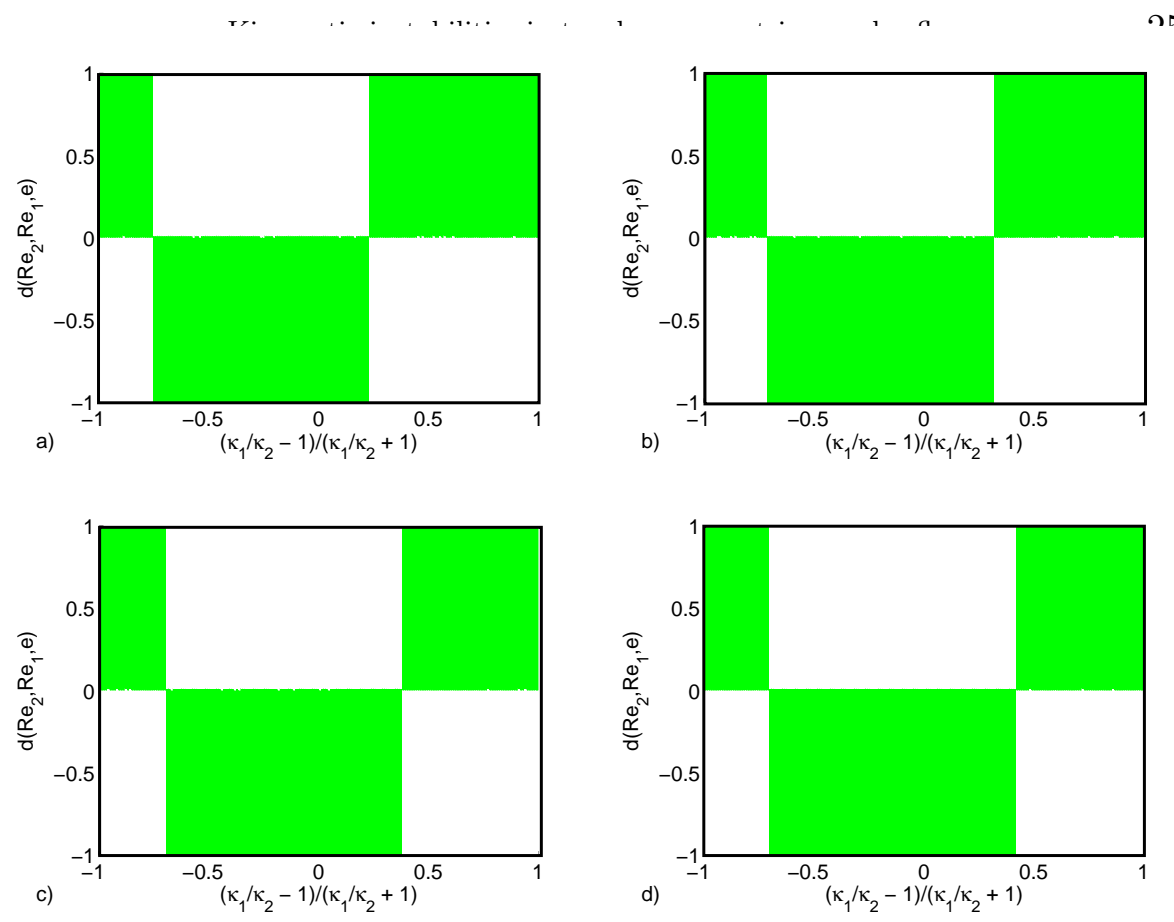

Figure 8. Regions of marginal stability, long wavelength approximation: $\beta=0$, $\varphi_{b}^{*}=1, \phi_{i, 0}=0.5, e=0.1, m_{2}=1, B_{1}=B_{2}=0$ : a) $m_{1}=4 / 3$; b) $m_{1}=2$; c) $m_{1}=3$; d) $m_{1}=5$; unstable - white, stable - green (light grey).

may occur. This ambiguity highlights the value of quick and relatively simple predictive methods such as here.

We now look at horizontal wells, which represent the other directional extreme of the buoyancy force. For the same net buoyancy force, Figs. 10-13 show the analogous results to Figs. 6-9, but with $\beta=\pi / 2$. Evidently, with a positive buoyancy gradient $\varphi_{b}^{*}=1$, the horizontal flow is strongly stabilized. Figs. $10 \& 11$ indicate that making the fluid on the narrow side of the annulus increasingly shear-thinning, (i.e. increasing $m_{2}$ ), apparently decreases the flow stability. Note that the parameters in the lower right quadrant are physically difficult to achieve. A similar destabilisation is observed at low eccentricity with increasing $m_{1}$, see Fig. 12. At larger eccentricities the overall stability of the flow is increased, see Fig. 12, and we see no evidence of destabilisation with increasing $m_{1}$.

Use of the long wavelength approximation is clearly efficient computationally and the plots in Figs. 6-13 are probably the clearest way of presenting the long wavelength stability. Although convenient, two problems with the long wavelength analysis are: (i) the long wavelength limit does not always predict the instability of the full problem, nor the maximal growth rates; (ii) the dimensionless variables that 

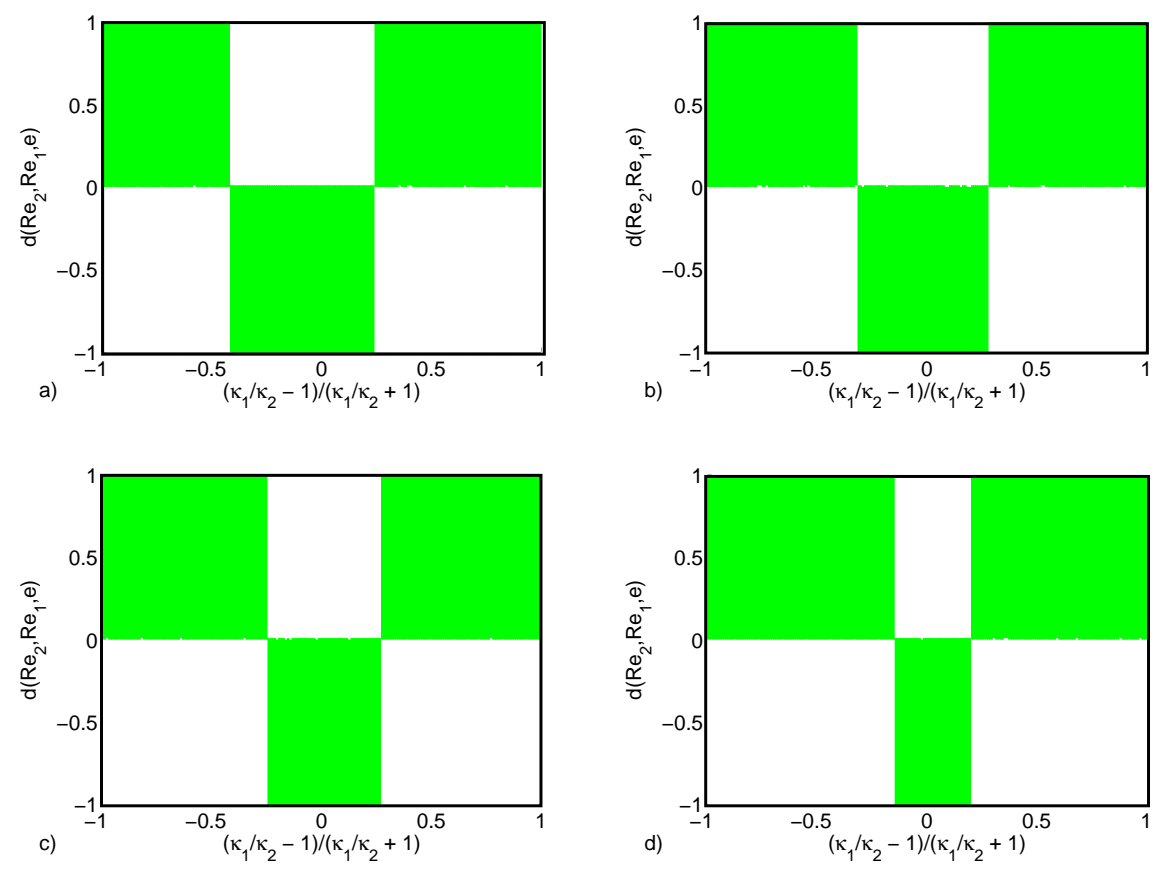

Figure 9. As Fig. 8 with $e=0.5$.
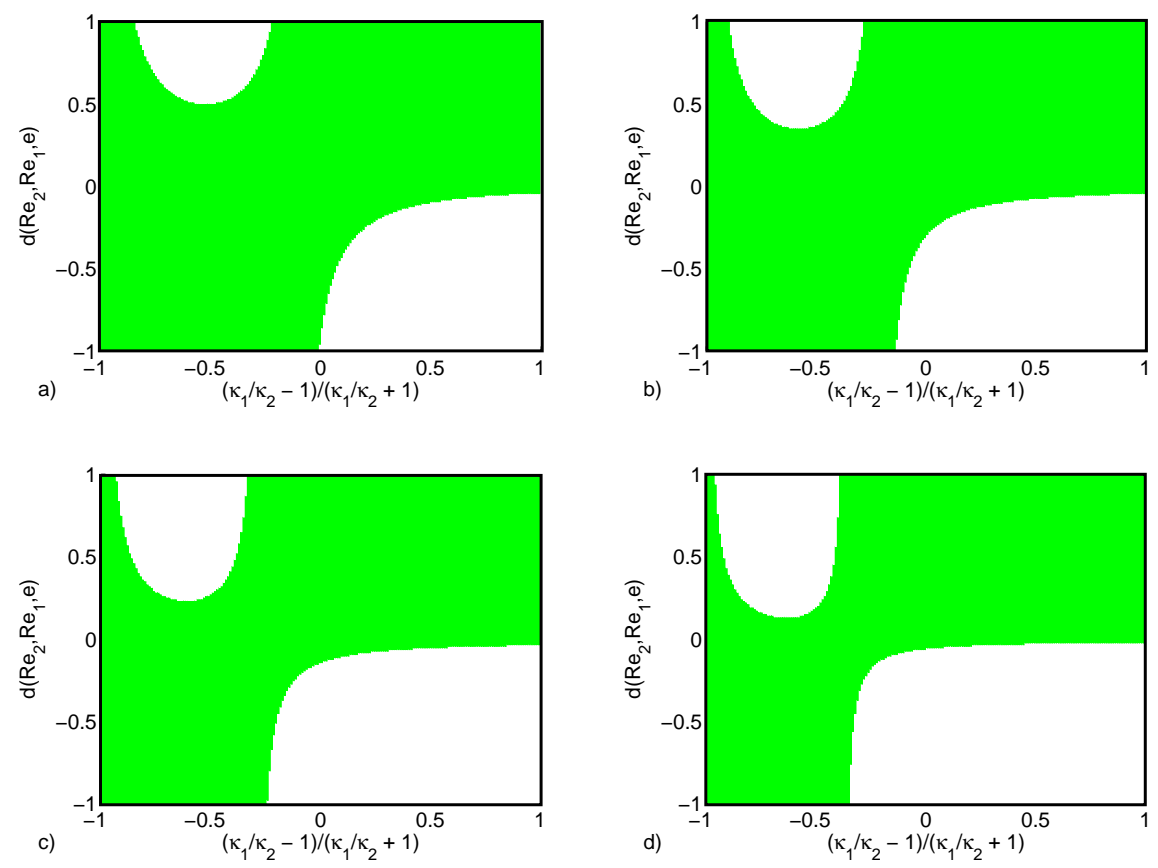

Figure 10. As Fig. 6 with $\beta=\pi / 2$. 

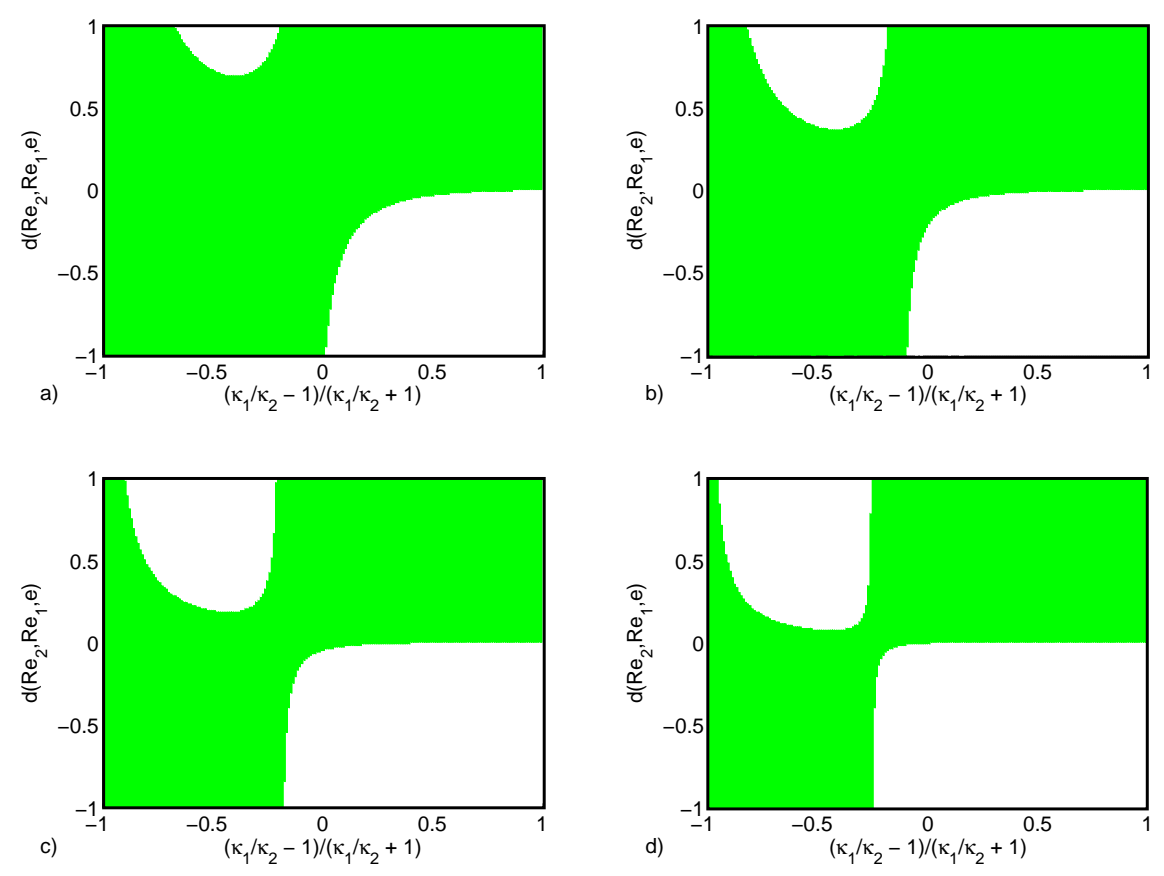

Figure 11. As Fig. 7 with $\beta=\pi / 2$.
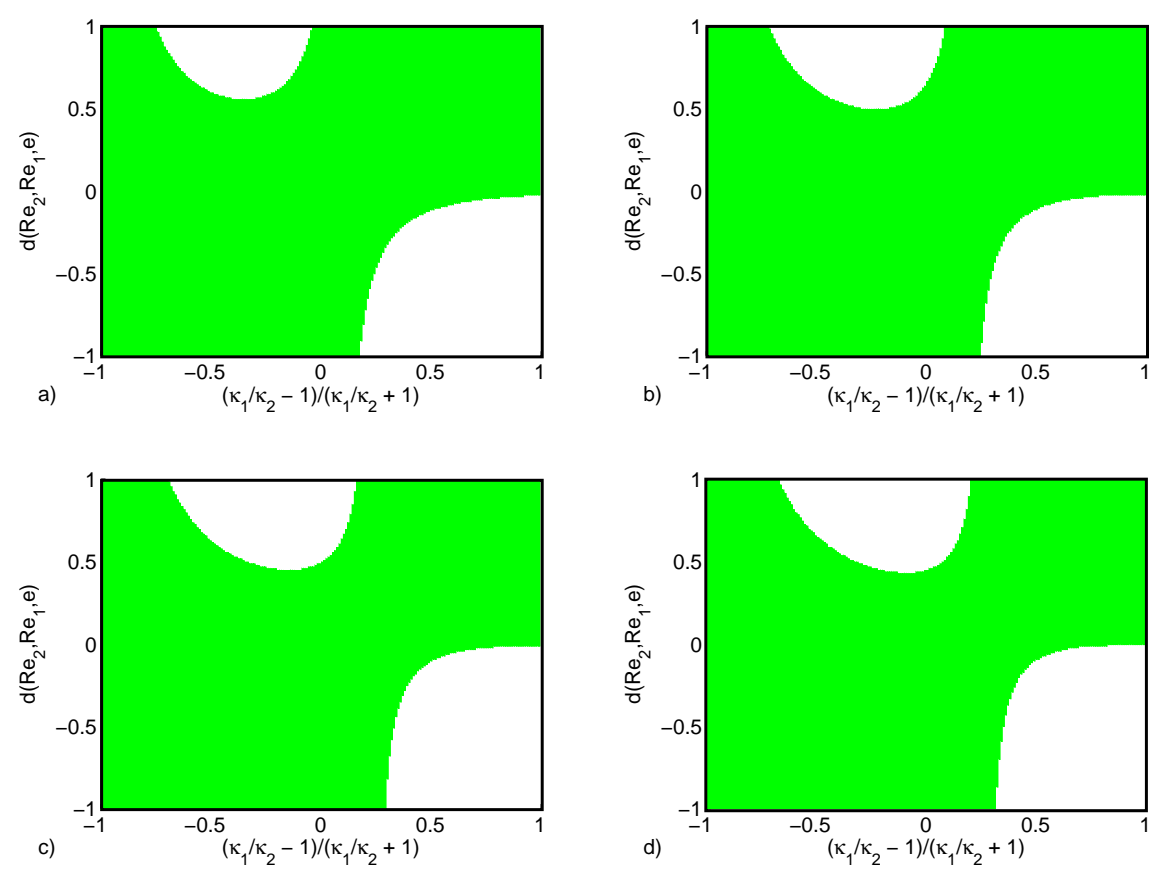

Figure 12. As Fig. 8 with $\beta=\pi / 2$. 

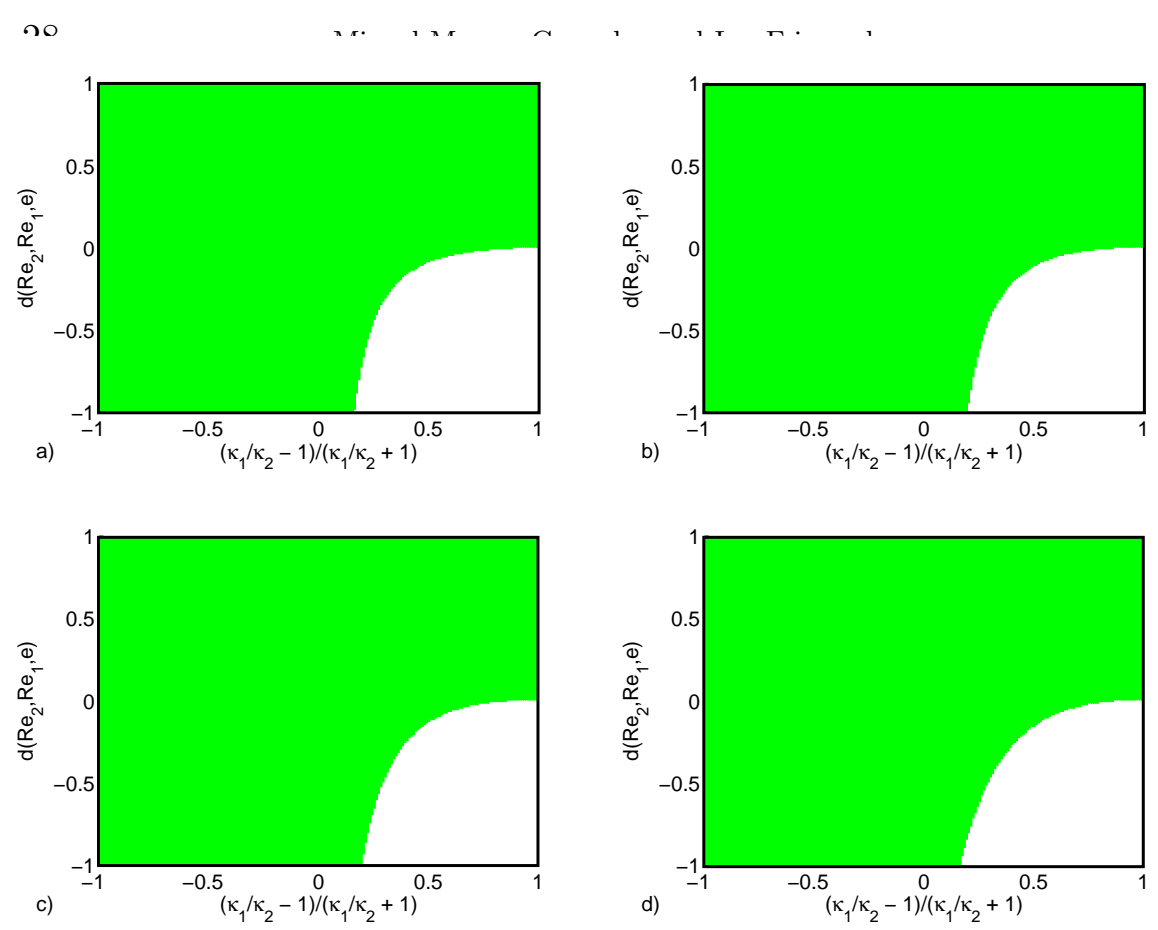

Figure 13. As Fig. 9 with $\beta=\pi / 2$.

are convenient for the long wavelength limit are not those useful for practical intuition. We illustrate both problems below, by solving the full stability system for example parameters.

We fix base parameters $\beta=0, e=0, \varphi_{\kappa}=2.5, \varphi_{b}^{*}=1, \phi_{i, 0}=0.5$, $B_{1}=0, B_{2}=0, \operatorname{Re}_{1}=1$ and $\operatorname{Re}_{2}=0.5$, (see Fig. 5a), and explore variation of the maximal eigenvalues with $m_{1}$ and $m_{2}$. In Fig. 14, we fix $m_{2}=1$ and take $m_{1}=4 / 3,2,3,5$. At $m_{1}=1$ (Newtonian case) the flow is unstable and as we increase $m_{1}$ the flow remains unstable for all values of $e$. Physically, we are making fluid 1 thinner/less viscous, so perhaps this is physically intuitive. As the wavenumber $\alpha$ increases $\lambda_{I, \max }$ changes smoothly until it asymptotes to the short wavelength limit as $\alpha \rightarrow \infty$. Although the long wavelength limit predicts the instability, the maximal growth rates are found in the short wavelength limit or at some intermediate wavenumber, see Fig. 14c \& d.

In Fig. 15, we fix $m_{1}=1$ and consider changes in $m_{2}=4 / 3,2,3,5$, for increasing $e$. A range of interesting behaviours are observed. First, the short wavelength and long wavelength limits clearly can give quite different stability predictions, as in Fig. 14. Second, we observe regions in which $\lambda_{I, \max }$ are apparently constant. Thirdly, for small values of $\alpha$, as we increase the power law index of fluid 2, we jump from an unstable flow to a stable flow, see e.g. $m_{2}=3$ and $m_{2}=5$. 

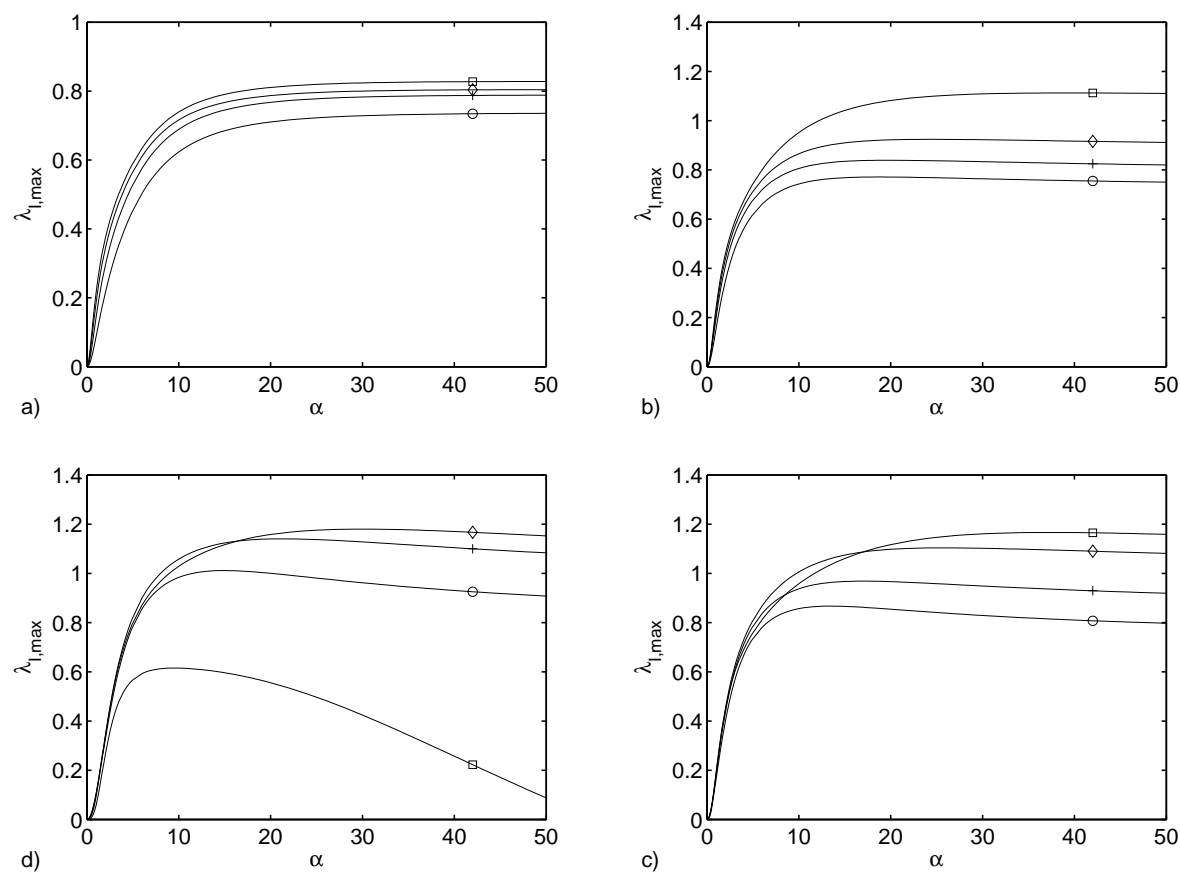

Figure 14. Examples of typical variations in least stable eigenvalue with wavenumber, $\beta=0, \phi_{i, 0}=0.5, \varphi_{b}^{*}=1, \operatorname{Re}_{1}=1, \operatorname{Re}_{2}=0.5, \varphi_{k}=2.5, B_{1}=B_{2}=0, m_{2}=1$; a) $e=0.1$; b) $e=0.3$; c) $e=0.5$; d) $e=0.8$. In each figure $m_{1}=4 / 3$ - marked with ○, $m_{1}=2$ - marked with,$+ m_{1}=3$ - marked with $\diamond, m_{1}=5$ - marked with $\square$.

The regions of near-constant $\lambda_{I, \max }$ are explained in part $1,[1]$. Essentially these regions are found when the interfacial modes become more stable than the viscous modes. The most unstable part of the viscous spectra turns about to be relatively insensitive to variations in wavenumber $\alpha$.

The jumping from stable to unstable at first appears strange, but is easily explained in the context of the long wavelength approximation. Here, at fixed $\beta$, stability is governed by the product $\left(W_{i, 2}-\right.$ $\left.W_{i, 1}\right) d\left(\operatorname{Re}_{2}, \operatorname{Re}_{1}, e\right)$. As we increase any one rheological parameter both the interfacial velocities and $d\left(\operatorname{Re}_{2}, \mathrm{Re}_{1}, e\right)$ will change in a nonlinear fashion, possibly changing time. It is this switching that leads to non-intuitive variations in stability. Figure 16 , plots $W_{i, 2}-W_{i, 1}$ and $d\left(\operatorname{Re}_{2}, \operatorname{Re}_{1}, e\right)$ for varying $m_{k}$, relative to the parameters in Fig. $14 \&$ 15. In Figs. 16a \& b we fix $m_{2}=1$ and vary $m_{1}$. We can see that $W_{i, 2}-W_{i, 1}$ and $d\left(\operatorname{Re}_{2}, \operatorname{Re}_{1}, e\right)$ have opposite sign and therefore the flow remains unstable. In Figs. $16 \mathrm{c} \& \mathrm{~d}$ we fix $m_{1}=1$ and vary $m_{2}$. We can see that $W_{i, 2}-W_{i, 1}$ is always positive (fluid two is always thinner) and $d\left(\operatorname{Re}_{2}, \mathrm{Re}_{1}, e\right)$ changes sign from negative to positive for increasing 

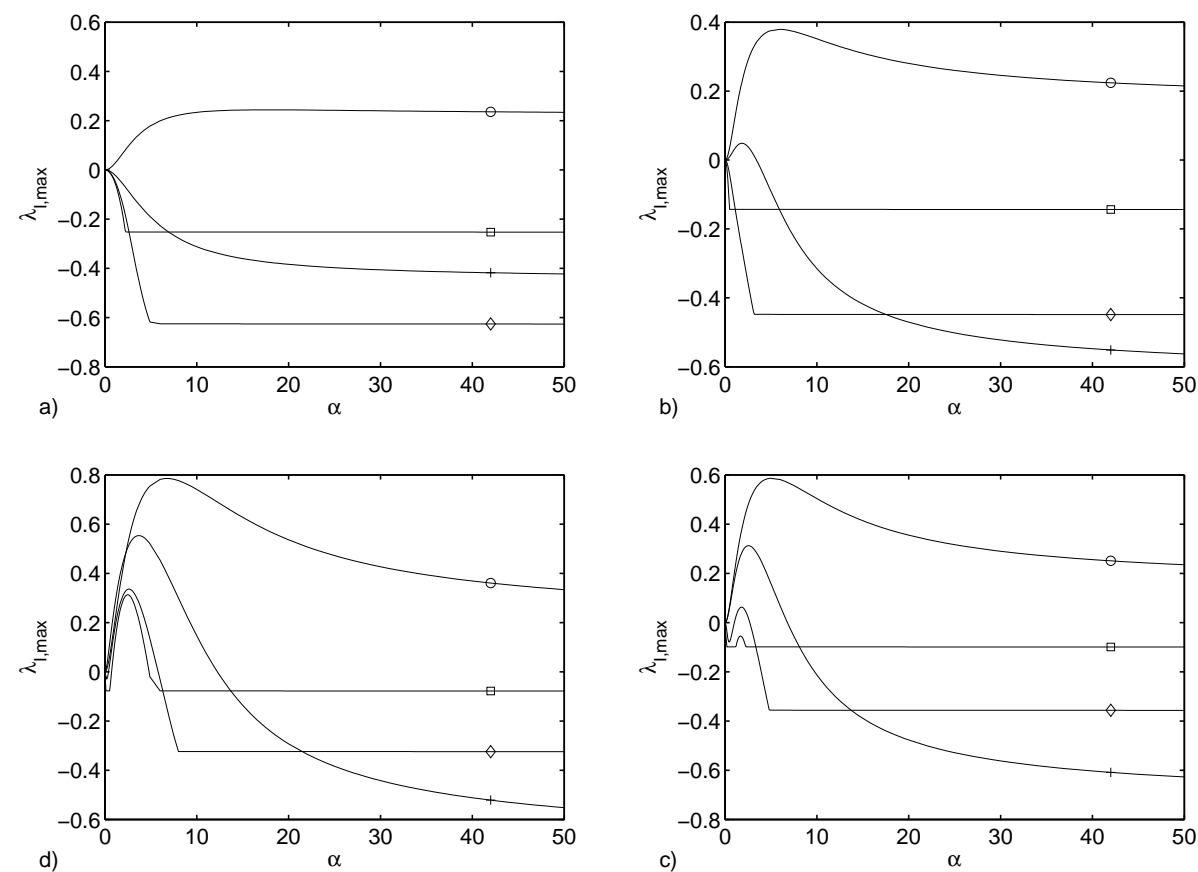

Figure 15. Examples of typical variations in least stable eigenvalue with wavenumber, $\beta=0, \phi_{i, 0}=0.5, \varphi_{b}^{*}=1, \operatorname{Re}_{1}=1, \operatorname{Re}_{2}=0.5, \varphi_{k}=2.5, B_{1}=B_{2}=0, m_{1}=1$; a) $e=0.1$; b) $e=0.3$; c) $e=0.5$; d) $e=0.8$. In each figure $m_{2}=4 / 3$ - marked with $\circ, m_{2}=2$ - marked with,$+ m_{2}=3$ - marked with $\diamond, m_{2}=5$ - marked with $\square$.

$m_{2}$, thus the flow will stabilize for large values of $m_{2}$. As we increase eccentricity the value of $m_{2}$ for which $d\left(\operatorname{Re}_{2}, \operatorname{Re}_{1}, e\right)$ changes sign increases. It is not possible to give a complete picture of even general rules regarding instability in the long wavelength limit. However, only simple quadrature is needed to evaluate the expression (60).

\subsection{Yield STRESS EFFECTS}

We now consider yield stress fluids, which are themselves shear-thinning. We can anticipate a similar level of complexity as for the power-law fluids in the above section. We therefore do not attempt to give a broad parametric study, but instead focus on those phenomena that are markedly different for yield stress fluids. As discussed in $\S 4.2$, certain parameter combinations allow static channels to develop in the base flow and the majority of these flows are linearly stable. The occurrence of static channels is intuitively governed by the yield stresses of the fluids and width of the annular gap, i.e. by eccentricity and azimuthal position. 

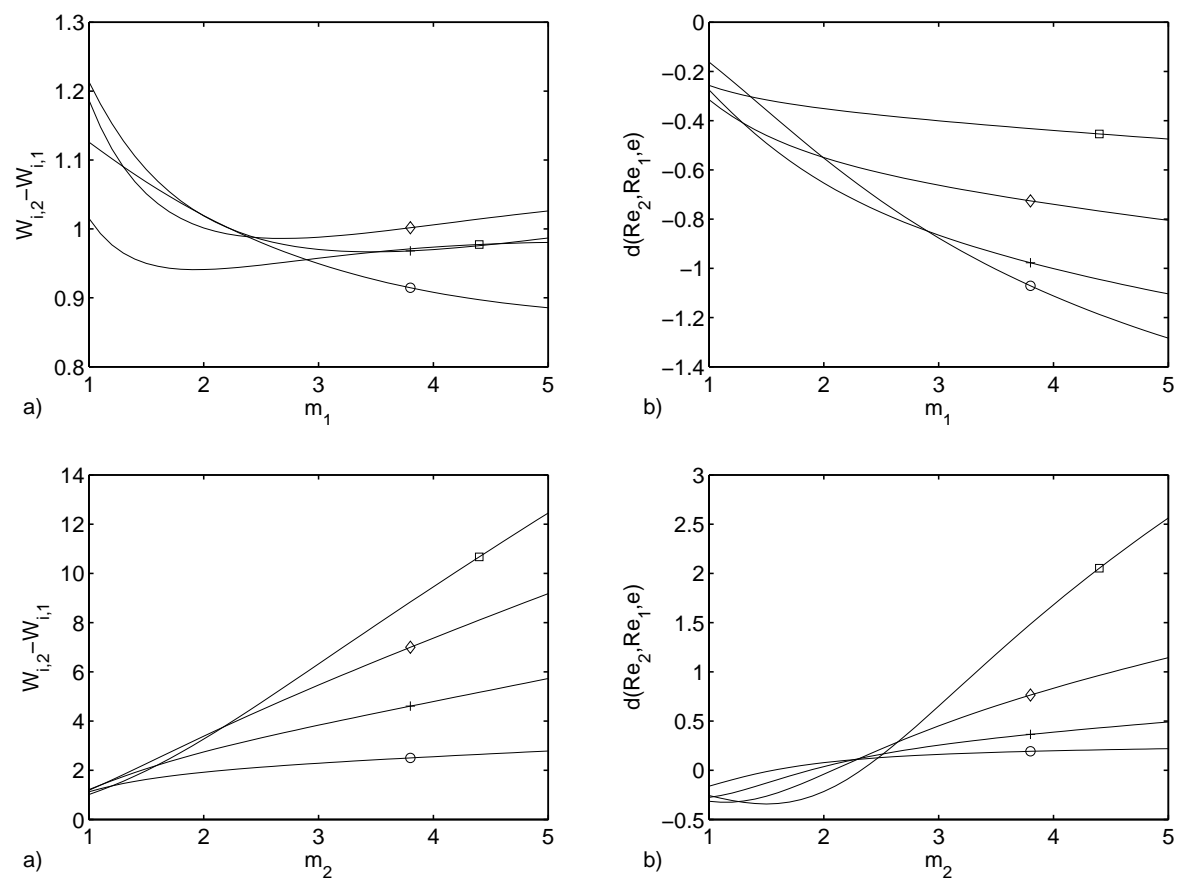

Figure 16. Examples of typical variations for $W_{i, 2}-W_{i, 1}$ and $d\left(\operatorname{Re}_{2}, \operatorname{Re}_{1}, e\right)$ for varying $m_{k}: \beta=0, \phi_{i, 0}=0.5, \varphi_{b}^{*}=1, \operatorname{Re}_{1}=1, \operatorname{Re}_{2}=0.5, \varphi_{k}=2.5, B_{1}=B_{2}=0$; a) and b) $m_{2}=1$; c) and d) $m_{1}=1$. In each figure $e=0.1$ - marked with $\circ, e=0.3$ - marked with,$+ e=0.5$ - marked with $\diamond, e=0.8$ - marked with $\square$.

By way of illustration, below in Fig. 17 we show predictions of the long wavelength theory (60), at different azimuthal positions in a vertical annulus with $e=0.5$. Fig. 18 shows analogous results in a horizontal annulus. What can be observed in Fig. 17 is that the usual checkerboard of stable and unstable regions is interrupted by large parameter regions where static channels are found in the base flow. Note that the base flow is independent of the Reynolds numbers. These same regions are found also in the horizontal annulus. Perhaps more interesting is that we see that there a direct transition from regions that are unstable, according to (60), to those with a static channel. This is a new type of transition and one that we should understand further.

Exploration of the spectrum of the full problem reveals that the addition of a yield stress appears to stabilise the flow by acting first on the short wavelength limit. Thus, as the yield stress is increased instability is found eventually only in an increasingly small interval of wavenumbers close to $\alpha=0$. An illustration of this is given in Fig. 19. Further increase of the fluid 1 yield stresses for the example in Fig. 19 results in the disappearance of the unstable interval and eventually the 

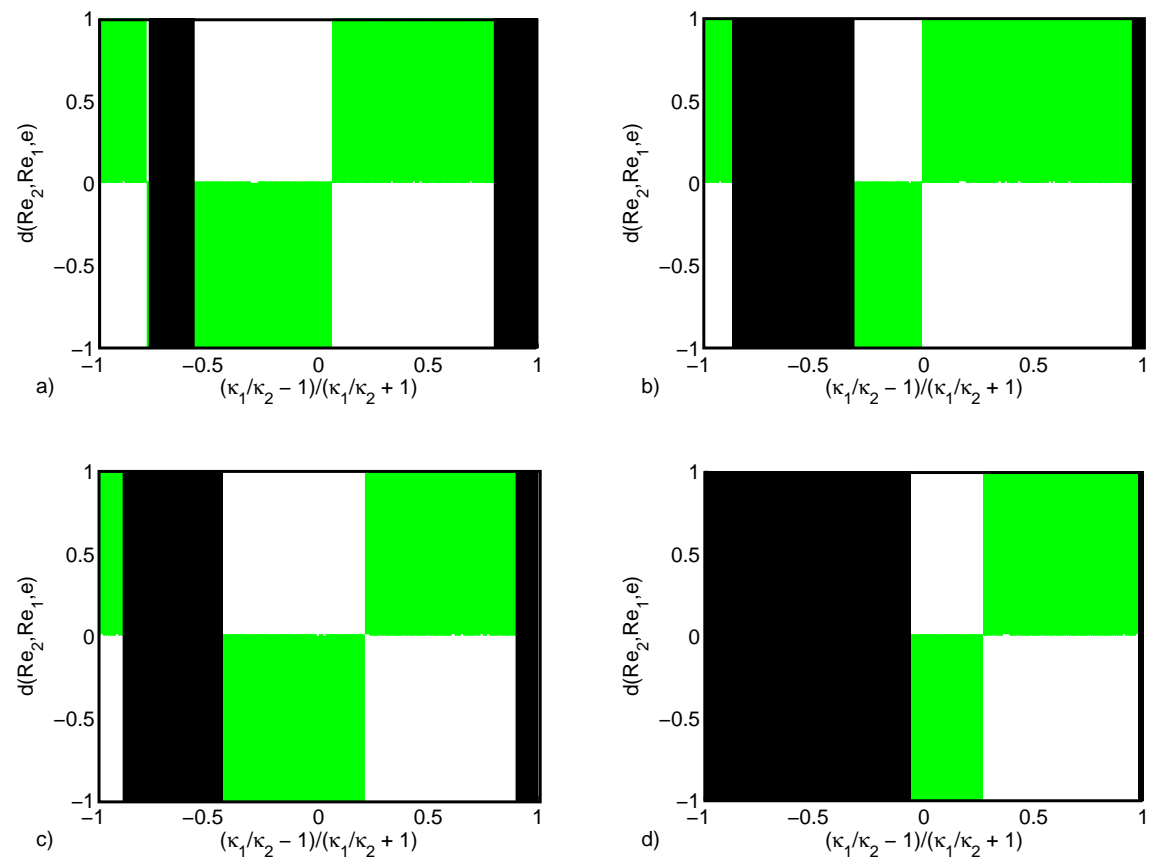

Figure 17. Regions of marginal stability, long wavelength approximation: $\beta=0$, $\varphi_{b}^{*}=1, e=0.5, m_{1}=m_{2}=1$ : a) $\phi_{i, 0}=0.25, B_{1}=1, B_{2}=0.5$; b) $\phi_{i, 0}=0.75$, $B_{1}=1, B_{2}=0.5$; c) $\phi_{i, 0}=0.25, B_{1}=0.5, B_{2}=1$; d) $\phi_{i, 0}=0.75, B_{1}=0.5, B_{2}=1$; unstable - white, stable - green (light grey), unyielded/static channel (black).

fluid 1 layer becomes static. This transition is characteristic of any of the transitions from stable to static regimes in Figs. 17-18.

A different transition occurs directly from unstable to static channel regimes. We illustrate this below in Fig. 20. For parameters: $\beta=0$, $\varphi_{b}^{*}=1, \operatorname{Re}_{1}=1, \operatorname{Re}_{2}=0.5, \varphi_{k}=2.5, B_{1}=0.1, B_{2}=1, m_{1}=m_{2}=1$, $\phi_{i, 0}=0.8$, we approach the static channel regime by progressively increasing the eccentricity, $e$. We present the evolution of the least stable eigenvalues of system (43)-(49) for $e \in[0.3,0.9]$ in Fig. 20a. The viscous spectrum remains fairly fixed, stabilising slightly. The main change is observed in the interfacial mode. As the static channel is approached, $\lambda_{I, \max } \rightarrow 0$ from above, but also $\lambda_{R, \max } \rightarrow 0$. We have presented only results for $\alpha=1$, although other wavenumbers are similar. Fig. 20b shows the base velocity solution for $e=0.3$ and $e=0.9$. For $e=0.9$ the fluid 2 layer is nearly static, with $\left|\Psi_{2,0, \phi}\right| \sim O\left(10^{-} 6\right)$.

The approach to the static layer flow is easier to understand via analysis of the long wavelength limit (60). From the results in [10] we know that $\chi_{k}(|\nabla \Psi|) \sim|\nabla \Psi|^{n_{k} /\left(n_{k}+1\right)}$ as $|\nabla \Psi| \rightarrow 0$. Thus, $\chi_{k}^{\prime}(|\nabla \Psi|) \sim$ $|\nabla \Psi|^{-1 /\left(n_{k}+1\right)}$ as $|\nabla \Psi| \rightarrow 0$. This singular behaviour causes difficulties 

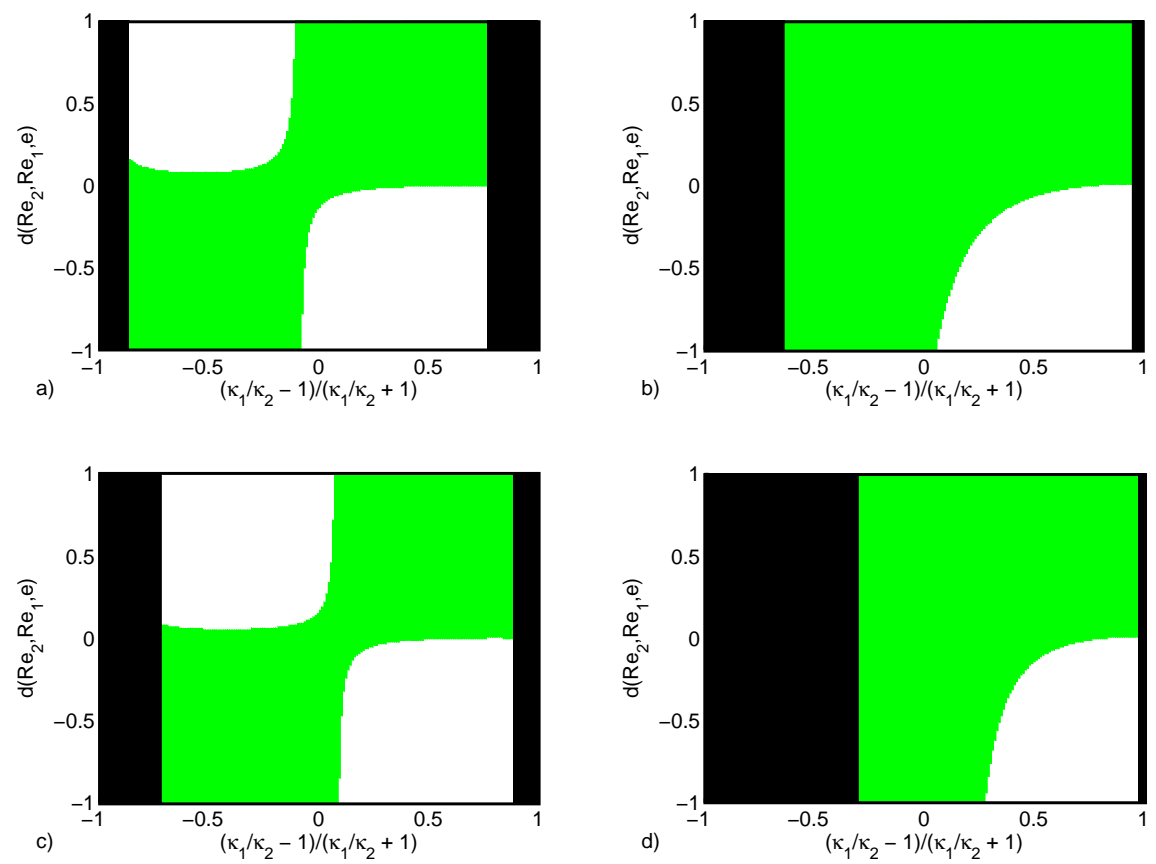

Figure 18. As Fig. 17 with $\beta=\pi / 2$.
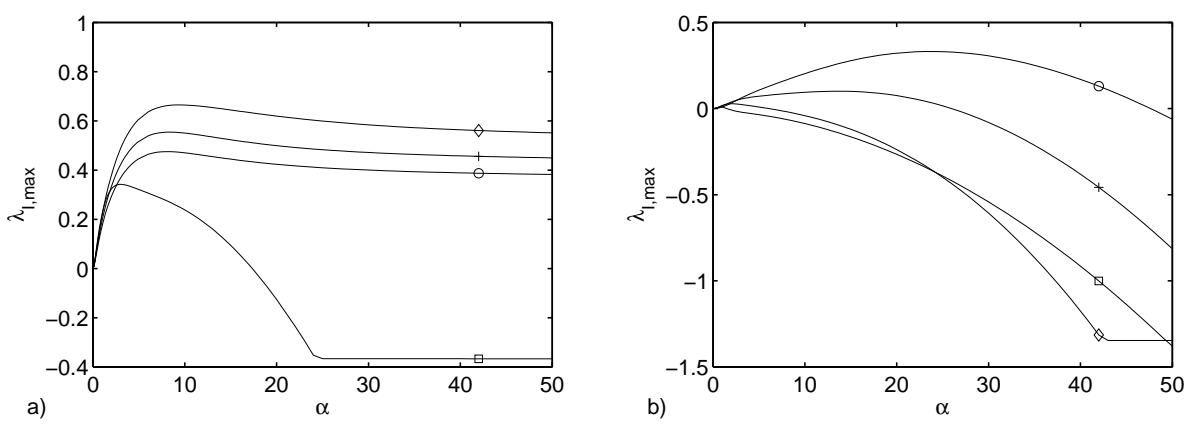

Figure 19. Examples of typical variations in least stable eigenvalue with wavenumber, $e=0.5, \beta=0, \varphi_{b}^{*}=1, \operatorname{Re}_{1}=1, \operatorname{Re}_{2}=0.5, \varphi_{k}=2.5, B_{2}=0.5, m_{1}=m_{2}=1$; a) $\phi_{i, 0}=0.25$; b) $\phi_{i, 0}=0.75$. In each figure $B_{1}=0.1$ - marked with $\circ, B_{1}=0.5$ marked with,$+ B_{1}=1$ - marked with $\diamond, B_{1}=2$ - marked with $\square$.

for numerical solution of (43)-(49), and some numerical noise can be observed in the calculated $\lambda_{I \text {,max }}$ in Fig. 20a, as $e$ becomes large. From (60) and the asymptotic behaviour of $\chi_{k}^{\prime}$ we can resolve the leading order behaviour of $\lambda$ at $\alpha=0$ as the static channel is approached:

$$
\begin{gathered}
\lambda_{1} \sim O\left(\left|\Psi_{2,0, \phi}\right|^{1 /\left(n_{2}+1\right)}\right) \\
\lambda_{2} \sim O\left(\varphi_{b}^{*} \sin \beta\left|\Psi_{2,0, \phi}\right|^{1 /\left(n_{2}+1\right)}\right)+O\left(\left|\Psi_{2,0, \phi}\right|^{2 /\left(n_{2}+1\right)}\right) .
\end{gathered}
$$



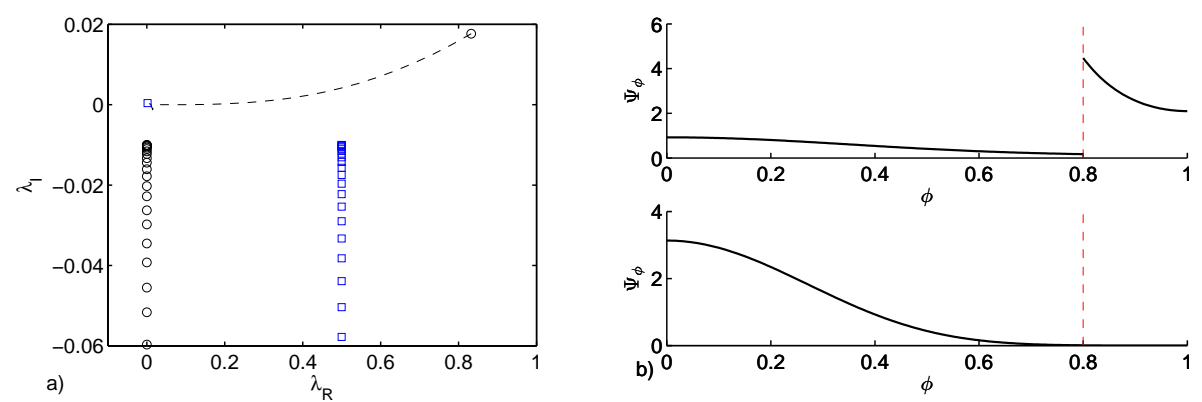

Figure 20. Transition from unstable to static channel for parameters: $\beta=0, \varphi_{b}^{*}=1$, $\operatorname{Re}_{1}=1, \operatorname{Re}_{2}=0.5, \varphi_{k}=2.5, B_{1}=0.1, B_{2}=1, m_{1}=m_{2}=1, \phi_{i, 0}=0.8$, with increasing eccentricity $e$. Figure a) shows the evolution of the least stable (interfacial) mode as $e$ is varied from $e=0.3$ (marked with $\circ$ ) to $e=0.9$ (marked with $\square$ ); see broken line. We also show the viscous spectrum at $e=0.3$ and $e=0.9$; that at $e=0.9$ (marked with $\square$ ) is offset along the real axis by 0.5 for clarity. Figure b) shows the base solution at $e=0.3$ and $e=0.9$.

The signs of the constants in the above limiting expression will be governed by the fluid 1 velocity profile, which remains $O(1)$ and in fact changes very little as fluid 2 becomes progressively static. Thus, the slightly strange transition direct from instability to the static channel is explained.

\section{Discussion}

This paper has considered a practically relevant generalisation of [1] to the case of shear-thinning and yield stress fluids, as are commonly used in primary cementing. Although the methodology is similar to the Newtonian case, there are a number of interesting differences in the results.

The occurrence of static mud channels in primary cementing has been known of since the 1960's, [2] and is a major cause of process failure. We have seen here that static channels can occur in either fluid layer, in the displaced or displacing fluid. It is those that occur on the narrow side of the annulus that are of most direct industrial relevance. Here we have quantified this phenomenon via the maximal volume of residual fluid that is possible to be left behind in the annulus, $f_{\text {static }}$. We have shown that $f_{\text {static }}$ depends on five dimensionless parameters and have presented a range of results that illustrate this dependency. In general $f_{\text {static }}$ is quick to compute as the solution of a simple nonlinear equation.

Via dimensional analysis, we have shown that the base solutions depend on a minimal set of 8 dimensionless parameters, which is 4 
more than the Newtonian fluid case in part 1. These additional parameters are the two power law indices and two Bingham numbers. These additional parameters thus capture the shear-thinning and yield stress effects. The stability problem depends on an additional 3 dimensionless parameters: two Reynolds numbers and a wavelength.

A range of analytical and semi-analytical results have been derived. For the industrially problematic static channel flows, we show that 3 of the 4 different types of static channel flows are linearly stable. The fourth type of static channel flow may be stable or unstable, this involves simply a restriction of the yielded channel width, but the interface has yielded fluid on both sides.

The most useful semi-analytical results certainly come from the long wavelength analysis. Here we have provided an equivalent characterisation of the stability to that in [1] for the Newtonian fluids. By this we mean that the final formulae for the eigenvalue contain equivalent terms to those in [1], albeit modified in a nonlinear fashion to account for the fluids. The formulae may be simply evaluated once the base flow is calculated and hence are suitable for rapid computation, e.g. as part of a process optimisation. In a similar way to the Newtonian fluid case, in the absence of buoyancy instability is governed by a product between the difference in interfacial velocities and a weighted difference in Reynolds number for the two fluids. The switching of signs of the terms in this product is responsible for much of the complexity that we observe in the results. When density differences are included the stability is changed in a fairly predictable way.

For power law fluids, i.e. only with shear-thinning effects, for vertical annuli we can make no simple statements regarding stability. For horizontal annuli with $\varphi_{b}>0$ the flow is stabilised. As the fluids become more shear-thinning the stability is slightly reduced.

Addition of a yield stress has the general effect of suppressing short wavelength instabilities. We also have the phenomenon of static channels, described above, which are commonly stable. Thus, our parametric results exhibit an additional two types of transition, from stable to static channel and direct from unstable to static channel. The latter is of course quite surprising and we have investigated the transition in detail, showing that the growth rates can indeed approach zero from above as the fluid channel solidifies.

In terms of practical matters, the most relevant fact is that static channels with an unyielded interface are linearly stable. This tends to suggest that ideas related to flow pulsation and the instigation of instability may not destabilise the mud channels. In short, the formation of static mud channels needs to be avoided as once formed they are likely to persist. 
An area that we have not touched here is which base parameters are likely to lead to multi-layer flows of the type studied here. Multilayer flows only arise from an ineffective underlying displacement for which the interface elongates into a pseudo-parallel flow; see Fig. 1f. Many parameter regimes lead to displacements that are in fact effective, i.e. steady traveling waves, see $[9,10,11]$. The analysis in [8] provides one method for predicting whether displacements will be steady or unsteady. Ideally, for practical application of the results in this paper we need to couple the predictions of a model such as in [8], with those from our work here, i.e. there is no point to consider the stability of a flow that never evolves in the first place.

\section{Acknowledgements}

This research has been carried out at the University of British Columbia, supported financially by Schlumberger and NSERC. We are grateful for this sponsorship.

\section{References}

1. M.A. Moyers-González \& I.A. Frigaard (2007) Kinematic instabilities in twolayer eccentric annular flows, part 1: Newtonian fluids. J Engng Math., DOI 10.1007/s10665-007-9178-y.

2. R.H. McLean, C.W. Manry and W.W. Whitaker (1966) Displacement Mechanics in Primary Cementing. Society of Petroleum Engineers paper number SPE 1488

3. M.J. Economides (1990) Implications of Cementing on Well Performance. In: E. B. Nelson (ed.) Well Cementing. Schlumberger Educational Services

4. Primary and Remedial Cementing Guidelines. Drilling and Completions Committee, Alberta, April 1995. Distributed by the Petroleum Industry Training Service.

5. E. B. Nelson (ed.) (2001) Well Cementing. Schlumberger Educational Services.

6. D. Guillot, H. Hendriks, F. Callet and B. Vidick (1990) Mud Removal. In: E. B. Nelson (ed.) Well Cementing. Schlumberger Educational Services

7. C.F. Lockyear, D.F. Ryan and M.M. Gunningham (1989) Cement Channelling: How to Predict and Prevent. Society of Petroleum Engineers paper number SPE 19865

8. S. Pelipenko \& I.A. Frigaard (2004) Visco-plastic fluid displacements in nearvertical narrow eccentric annuli: prediction of travelling wave solutions and interfacial instability. J Fluid Mech, 520: 343-377

9. S.H. Bittleston, J. Ferguson and I.A. Frigaard (2002) Mud removal and cement placement during primary cementing of an oil well; laminar non-Newtonian displacements in an eccentric annular Hele-Shaw cell. J Engng Math 43: 229-253

10. S. Pelipenko and I.A. Frigaard (2004) On steady state displacements in primary cementing of an oil well. J Engng Math, 46(1): 1-26

11. S. Pelipenko \& I.A. Frigaard (2004) Two-dimensional computational simulation of eccentric annular cementing displacements. IMA J Appl Math, 64(6): 557-583 
12. A. Tehrani, J. Ferguson and S.H. Bittleston (1992) Laminar Displacement in Annuli: A Combined Experimental and Theoretical Study. Society of Petroleum Engineers paper number SPE 24569

13. A. Tehrani, S.H. Bittleston and P.J.G. Long (1993) Flow instabilities during annular displacement of one non-Newtonian fluid by another. Experiments in Fluids 14: 246-256

14. M.A. Moyers-González (2006) Transient Effects in Oilfield Cementing flows. Ph.D. dissertation, University of British Columbia

15. M.A. Moyers-González, I.A. Frigaard, O. Scherzer \& T.-P. Tsai (2007) Transient effects in oilfield cementing flows: qualitative behaviour. Euro J Appl Math., 18: 477-512.

16. P. J. Schmid, D. S. Henningson (2001) Stability and Transition in Shear Flows. Springer-Verlag, New York, Inc.

17. I. A. Frigaard, S. D. Howison and I.J. Sobey (1994) On the stability of Poiseuille flow of a Bingham Fluid. J. Fluid Mech., 263: 133-150. 
stabvpfld.tex; 11/04/2008; 14:43; p.38 